ARTICLE

\title{
Maintenance of quiescent oocytes by noradrenergic signals
}

\author{
Jeongho Kim ${ }^{1,6}$, Moonjung Hyun (10) 2,4,6, Masahiko Hibi (i] ${ }^{3}$ \& Young-Jai You $2,3,5 凶$
}

\begin{abstract}
All females adopt an evolutionary conserved reproduction strategy; under unfavorable conditions such as scarcity of food or mates, oocytes remain quiescent. However, the signals to maintain oocyte quiescence are largely unknown. Here, we report that in four different species - Caenorhabditis elegans, Caenorhabditis remanei, Drosophila melanogaster, and Danio rerio - octopamine and norepinephrine play an essential role in maintaining oocyte quiescence. In the absence of mates, the oocytes of Caenorhabditis mutants lacking octopamine signaling fail to remain quiescent, but continue to divide and become polyploid. Upon starvation, the egg chambers of $D$. melanogaster mutants lacking octopamine signaling fail to remain at the previtellogenic stage, but grow to full-grown egg chambers. Upon starvation, $D$. rerio lacking norepinephrine fails to maintain a quiescent primordial follicle and activates an excessive number of primordial follicles. Our study reveals an evolutionarily conserved function of the noradrenergic signal in maintaining quiescent oocytes.
\end{abstract}

\footnotetext{
${ }^{1}$ Department of Biological Sciences, Inha University, Incheon 22212, South Korea. ${ }^{2}$ Department of Biochemistry and Molecular Biology, Virginia Commonwealth University, Richmond, VA 23298, USA. ${ }^{3}$ Graduate School of Science, Nagoya University, Nagoya 464-8602, Japan. ${ }^{4}$ Present address: Biological Resources Research Group, Bioenvironmental Science \& Toxicology Division, Korea Institute of Toxicology (KIT), Gyeongsangnam-do 52834, South Korea. ${ }^{5}$ Present address: Department of Internal Medicine, University of Texas Southwestern Medical Center, Dallas, TX 75390, USA. ${ }^{6}$ These authors contributed equally: Jeongho Kim, Moonjung Hyun. ${ }^{凶}$ email: Young-Jai.You@UTsouthwestern.edu
} 
S exual reproduction in animals requires timely fusion of an egg and a sperm; the right environment at the right timing in producing and releasing gametes are essential. While in spermatogenesis, meiosis and differentiation proceed continuously without cell cycle arrest, in oogenesis, meiosis is arrested and reinitiated upon signals in order to not waste eggs, which contain huge amounts of resources.

In mammals, the primordial oocytes produced at birth remain quiescent, arresting at diplotene of the meiotic prophase I, and can remain quiescent for as long as their entire reproductive span. At puberty, upon release of the gonadotropins follicle stimulating hormone (FSH) and luteinizing hormone $(\mathrm{LH})$, primordial follicles consisting of a meiotically arrested oocyte and surrounding granulosa cells undergo activation (termed PFA: primordial follicle activation), resume growth, and the oocytes complete meiosis I to fully mature.

The timing of oocyte awakening is the key to reproduction, not only by providing available oocytes with the right timing, but also by preserving the pool of available and high-quality oocytes. Indeed, the process is tightly regulated by coordinated mechanisms involving timely release of endocrine hormones, constant communications among granulosa cells and the oocyte via gap junctions and juxtacrine signals, and assessment of the nutritional state of the female. In mammals, a signal of natriuretic peptide precursor type C (NPPC) from granulosa cells is necessary for maintaining high cAMP concentration in oocytes, which is required to maintain meiotic arrest ${ }^{1,2}$. $\mathrm{LH}$ whose receptors are expressed only after secondary follicles grow to small antral follicles releases the oocyte from meiotic arrest by antagonizing the NPPC signal in granulosa cells ${ }^{3}$. The mTORC1 signaling, critical in linking the nutritional state of an animal to its cellular metabolism, plays an essential role in awakening quiescent oocytes. KIT ligand (KITL) secreted from the pre-granulosa cells upon mTORC1 activation binds to the KIT receptors in the primordial oocyte, which activates the PI3K-AKT pathway in the oocyte and results in cytoplasmic sequestration of FOXO3A, leading to PFA 4 . Reduced mTOR signaling prevents quiescent oocytes from awakening, whereas excessive mTOR signaling awakens all quiescent oocytes prematurely ${ }^{4}$.

The signals to maintain the quiescent primordial follicles, however, are still not fully understood. Anti-Müllerian hormone $(\mathrm{AMH})$, a member of the TGF $\beta$ superfamily produced by granulosa cells, inhibits the $\mathrm{PFA}^{5}$ and FSH-mediated steroidogenesis ${ }^{6}$. Female $\mathrm{Amh}^{-1-}$ mice carry a smaller number of primordial follicles as they age compared to the control, despite a normal appearance of the ovary and litter size ${ }^{7}$, suggesting loss of primordial follicles due to excess activation. The level of $\mathrm{AMH}$ is temporarily increased immediately after a PFA then falls, indicating that locally acting $\mathrm{AMH}$ prevents the upcoming PFA, limits the number of recruited primordial follicles for activation at a given time and maintains the quiescent oocyte reserve. However, when oocytes are released from the follicles into a culture medium in vitro, oocytes are spontaneously released from quiescence $^{8}$. Also, when fetal ovaries were severed from their nerves, isolated and cultured in a serum-free medium, the oocytes successfully underwent PFA in vitro ${ }^{9,10}$. These observations suggest the existence of signals besides $\mathrm{AMH}$ that are necessary for maintaining quiescence and inhibitory to PFA originated from outside of the ovary.

Zebrafish, a widely used small animal model, spawn once a year only during a monsoon in the wild ${ }^{11}$, indicating that the activation and the growth of primordial follicles is tied to availability of food. The growing oocytes are divided into five stages of primary growth (stage (I), cortical alveolus stage (II), vitellogenesis (III), maturation (IV), and mature egg (V)) based on size and morphology ${ }^{12}$. Quiescent oocytes arrested in meiosis prophase I are maintained at stage I. As in mammals, once awakened, the oocytes grow to stage IV by actions of FSH and $\mathrm{LH}^{12}$. The oocyte growth and maturation in zebrafish and mammals share several molecular mechanisms; the KITLs secreted from the granulosa cells act on the receptors residing in the oocytes, potentially linking nutrients signals such as insulin to the growth of oocytes. Although AMH does not play the same role in the fish as in mammals, other TGF $\beta$ family members such as inhibin, activin, and BMP15 play important roles in inhibiting and promoting the oocyte maturation mostly from stage II - IV by controlling the sensitivity to gonadotropins, as $\mathrm{AMH}$ does ${ }^{13}$. The signals to maintain quiescent oocytes, however, have not been identified.

The two most studied invertebrate models, D. melanogaster and C. elegans, also maintain quiescent oocytes. In D. melanogaster, oocyte quiescence can be induced by short photoperiod conditions at low temperature or starvation ${ }^{14}$. Flies at low temperature rarely feed, suggesting that starvation alone could be a sufficient cause of ovarian quiescence ${ }^{15}$. The fat body serves similar functions to those of the adipose tissue and the liver in vertebrates and regulates reproduction by nutritional status ${ }^{16}$. Under well-fed conditions, the fat body secrets Stunted (Sun), which binds to its receptor Methuselah (Mth) in the insulin producing cells (IPCs), which in turn promotes secretion of Drosophila Insulin-Like Peptides (dILPs) from the IPCs ${ }^{17}$. The high insulin signal prodūced in response to a good nutritional state promotes reproduction by (a) increasing the rate of cell division in the germline stem cells ${ }^{18}$ and by (b) increasing the level of juvenile hormone $(\mathrm{JH})$, whose activity is absolutely required for vitellogenesis beginning from the stage 8 egg chamber to accumulate yolk ${ }^{19}$. Upon binding to its receptors, Germ-cell-expressed (Gce) and Methoprene-tolerant (Met), JH upregulates the expression of vitellogenin receptors as well as the genes involved in vitellogenin synthesis ${ }^{20}$. When the fly is starved, the ovaries become quiescent and the levels of insulin and $\mathrm{JH}$ are reduced $^{21,22}$. However, whether reduction of insulin and $\mathrm{JH}$ is sufficient to maintain quiescent ovaries, or another inhibitory signal is necessary remains unknown.

C. elegans hermaphrodites are self-fertilizing and each produces $\sim 300$ sperm before the onset of oogenesis. C. elegans oogenesis occurs inside the gonadal sheath, which is functionally equivalent to somatic follicle cells in vertebrates and flies. Competent oocytes at the diakinesis stage (hereafter called diplotene stage) in meiosis are self-fertilized by sperm stored in the spermatheca at a constant rate. As the sperm are made and stored before oogenesis and are ready to fertilize as soon as an oocyte is ready, the second meiotic arrest at the metaphase present in most gonochoristic animals does not exist in C. elegans. The most proximal oocyte is activated by the sperm signal, and an oocyte matures every 23 min under well-fed conditions ${ }^{23}$. When sperm are exhausted, quiescent oocytes accumulate inside the gonadal sheath. The signal required to maintain quiescent oocytes in the absence of sperm has not been identified.

The adrenergic innervation of the ovary is thought to be required for modulation of the function of ovarian vasculature and ovarian sheath cells. Despite the reports from multiple animals that the ovaries are innervated by cells expressing norepinephrine in vertebrates or octopamine in invertebrates, the function of noradrenergic innervation of the ovary is unclear. In D. melanogaster, octopamine is required for the egg-laying process $^{24}$. However, C. elegans mutants lacking octopamine, and zebrafish and mouse mutants lacking norepinephrine reproduce normally ${ }^{25-27}$, indicating that the role of noradrenergic signaling in fertility might be hidden under well-fed laboratory conditions. Because of the well-known roles of noradrenergic signals in the responses to stresses such as the fight-or-flight response ${ }^{28}$ and to starvation $^{29}$, we investigated the function of norepinephrine and 


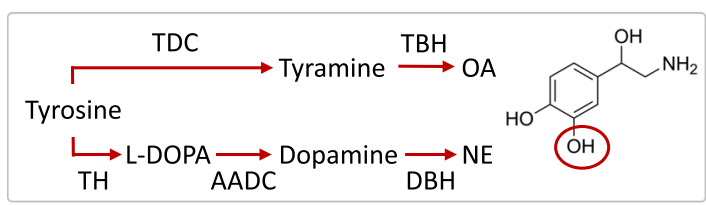

\section{b. C. elegans}
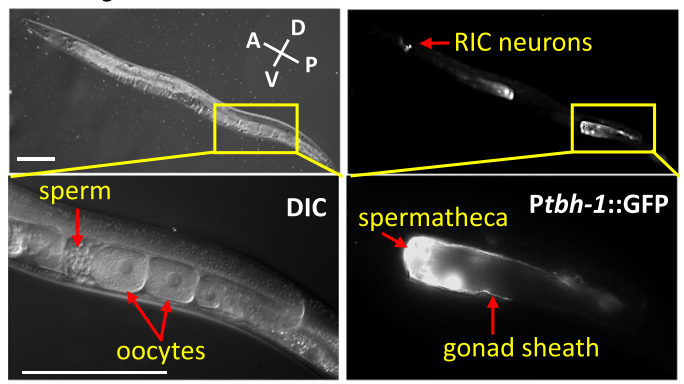

c. D. melanogaster
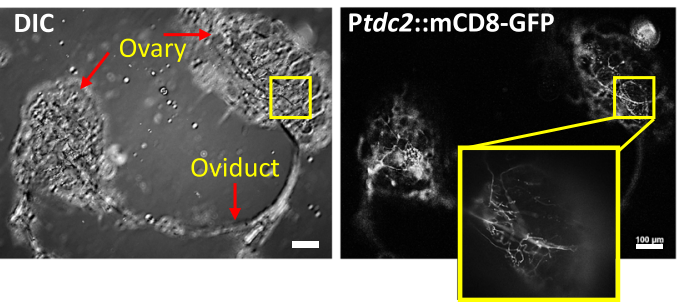

d. D. rerio (9-d starved)

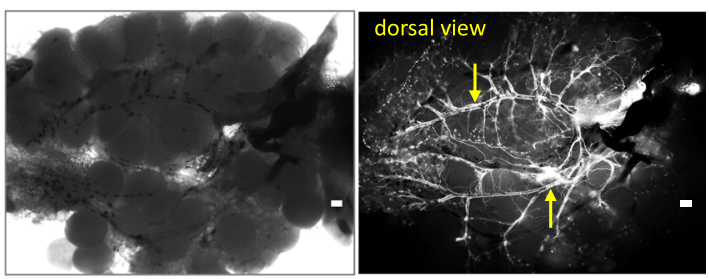

e. $D$. rerio (24-d starved)

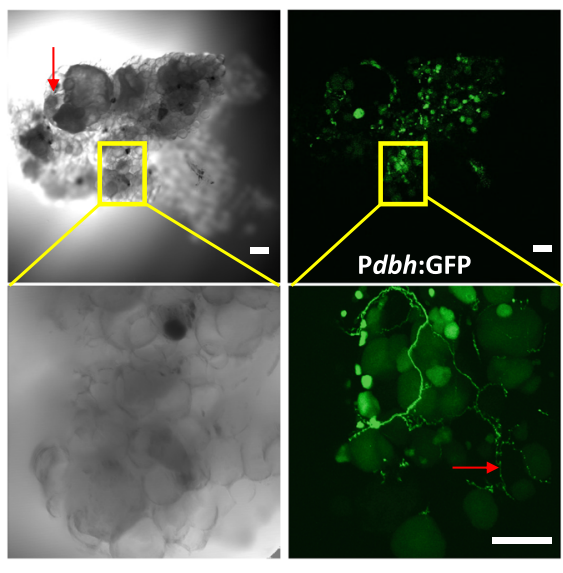

Fig. 1 Noradrenergic innervation of ovaries in the three animals. a The synthesis pathways of norepinephrine (NE) and octopamine (OA) and the enzymes for each step. TDC: Tyrosine decarboxylase, TBH: Tyramine $\beta$-hydroxylase, TH: Tyrosine hydroxylase, AADC: Aromatic L-amino acid decarboxylase, DBH: Dopamine $\beta$-hydroxylase, L-DOPA: L-Dihydroxyphenylalanine. NE is shown. The hydroxyl group (red circle) is absent in OA. b GFP

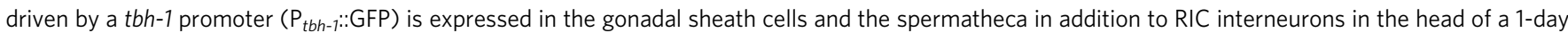
old adult $C$. elegans. All 15 C. elegans examined showed similar expression pattern. The left panel shows developing oocytes surrounded by gonadal sheath (top) and the enlarged image of the inset in a DIC image (bottom). The right panel shows the GFP expression of tbh-1. The highest expression is observed in the spermatheca and the proximal three pairs of the gonadal sheath cells that surround diakinesis-staged oocytes. GFP is also expressed in a pair of RIC neurons in the head. c The expression of a tdc2-GAL4 reporter with a membrane targeting mCD8-GFP marks OA-expressing ovarian neurons originated from the abdominal nerves in a virgin female $D$. melanogaster. The neuronal branches cover the entire surface of the ovary. Similar $t d c 2$-expression was observed from 18 flies. d EGFP driven by a $d b h$ promoter is expressed in $d b h$-expressing neurons that surround the entire surface of the ovary in $D$. rerio. An image from a light microscopy shows many mature and growing oocytes in the ovary of 9-day starved zebrafish. The dbh-expressing nerves run along the main vasculatures on the dorsal side of the ovary (arrows). Similar $d b h$-expression was observed from 8 fish. e DIC and confocal images of the ovary of a 23-d-starved zebrafish. Degenerating oocytes are shown (red arrow). A higher magnification image (bottom) shows small-sized varicosities along the neurons (red arrow). Similar dbh-expression at lower magnification was observed from four fish. b-e Scale bar $=100 \mu \mathrm{m}$.

octopamine in ovaries under unfavorable conditions, where the maintenance signal for oocyte quiescence will be manifest. Here, we show that noradrenergic signal is essential to maintain quiescent oocyte pools in all four model organisms of C. elegans, C. remanei, Drosophila and zebrafish under the stressful conditions, revealing a new role of noradrenergic signaling in safeguarding oocytes.

\section{Results}

Noradrenergic innervation of ovaries in the three animals. Norepinephrine (NE) and its invertebrate counterpart octopamine $(\mathrm{OA})$ are produced by tyrosine hydroxylase $(\mathrm{TH})+$ dopamine $\beta$ hydroxylase (DBH), and by tyrosine decarboxylase (TDC) + tyramine $\beta$ hydroxylase (TBH), respectively (Fig. 1a). Using transgenic animals carrying green fluorescent protein (GFP) fused with promoters of $t b h-1$ in C. elegans, $t d c 2$ in D. melanogaster, and $d b h$ in D. rerio, we observed that in all cases, the ovaries were heavily innervated by processes that express the enzymes (Fig. 1b-e). This suggests that in all three species, NE or OA are synthesized and released to the ovary.

In C. elegans, consistent with a previous report ${ }^{25}$, OA synthetic enzymes are highly expressed in the spermatheca and the proximal gonadal sheath (Fig. 1b). tbh-1 expression in the gonadal sheath begins from the late larval stage 4 (L4), when diplotene-stage oocytes appear, while $t b h-1$ expression in RIC interneurons in the head begins as early as at the L1 stage (RIC is indicated in Fig. 1b). This $t b h-1$ expression in gonadal sheath cells accounts for the observation that adult C. elegans produce at least five times more OA than larvae, which express OA only in $\mathrm{RIC}^{29}$. The level of TBH-1 is the strongest in the proximal three pairs of gonadal sheath cells, which cover the meiotic oocytes inwaiting, suggesting a potential OA function in oocyte quiescence. Strong expression in the spermatheca (see Fig. 2a, Sp.), which are involved in fertilizing and moving the fertilized eggs, suggests a role of $\mathrm{OA}$ in ovulation, as has been reported in D. melanogaster ${ }^{30}$.

In D. melanogaster, each ovary consists of $\sim 15$ sacs of ovarioles, which contain a series of developing egg chambers in an assembly-line fashion. Oogenesis begins in the germarium where germline stem cells reside. The stage- 2 egg chambers emerge from the germarium surrounded by a layer of somatic follicle cells. The egg chamber rapidly develops into the full-grown stage-14 oocyte under well-fed conditions. Each ovariole is surrounded by a single layer of contractile epithelium, the epithelial sheath, and then 
a

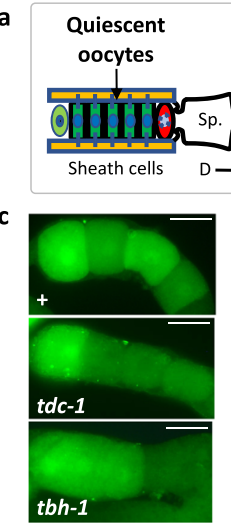

e

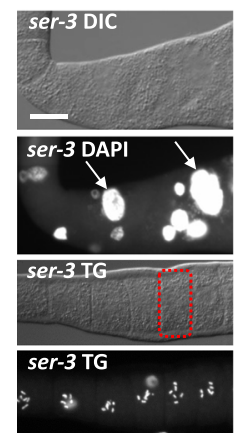

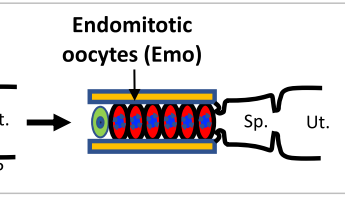

d

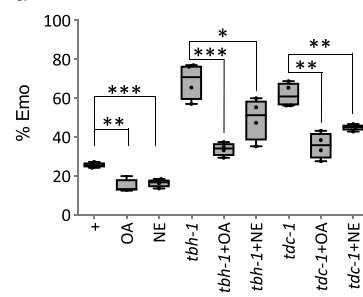

f

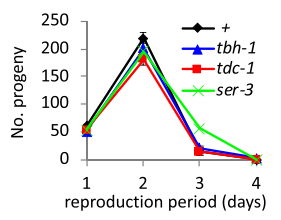

g

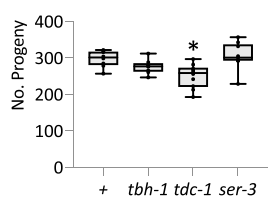

b

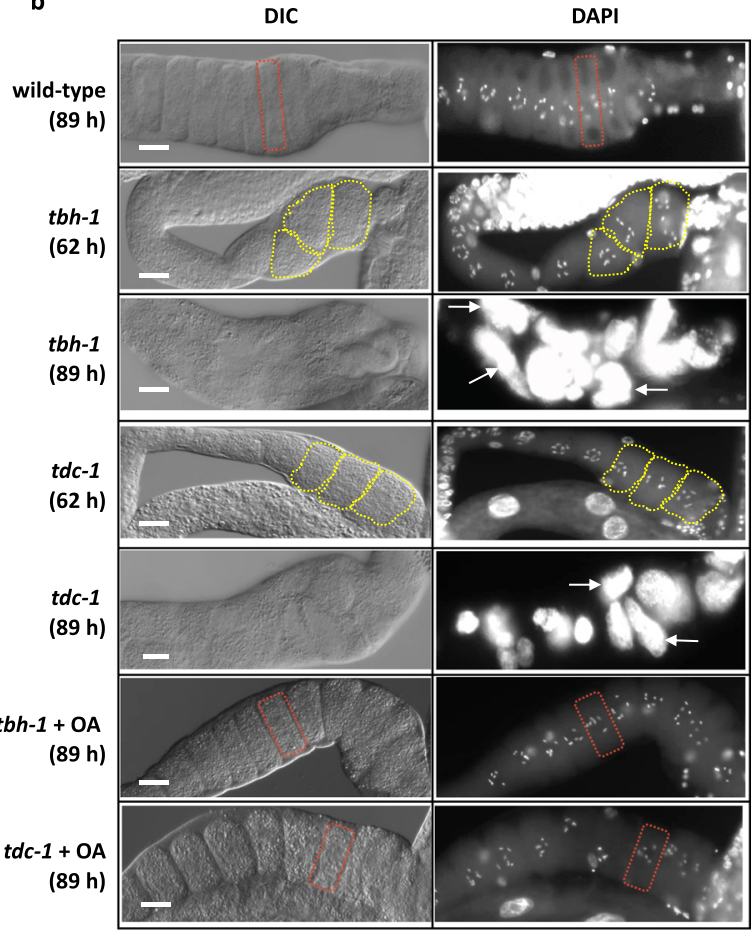

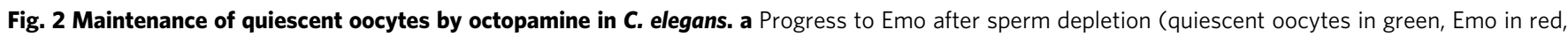
the gap junctions between oocytes and sheath cells in blue). D: distal, P: proximal, Sp.: Spermatheca, Ut.: Uterus. b Representative images of DIC (left) and DAPI staining (right) of oocytes (rectangles: quiescent oocytes, polygons: oocytes, arrows: Emo). The images represent 84 gonad images of wild-type, 9 gonads of $62 \mathrm{~h}$ of $t b h-1,5$ gonads of $t d c-1,94$ gonads of $89 \mathrm{~h}$ of $t b h-1,77$ gonads of $t d c-1,66$ gonads of $t b h-1+O A, 66$ gonads of $t d c-1+O A$. c

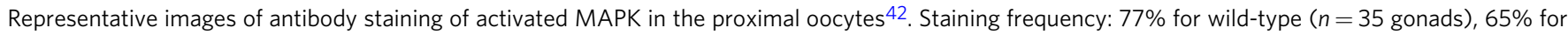
tdc- 1 ( $n=17$ gonads), and $70 \%$ for tbh-1 ( $n=30$ gonads). d Quantification of Emo at 89 h after hatch. Two-sided $t$-test: $p=0.001$ (wild-type ( $n=113$ animals) vs. wild-type + OA ( $n=99$ animals)), 0.0003 (wild-type vs. wild-type $+\mathrm{NE}(n=109$ animals)), $0.0004(t b h-1$ ( $n=132$ animals) vs. $t b h-1+0 A$ ( $n=99$ animals)), 0.035 (tbh-1 vs. tbh-1+NE ( $n=111$ animals)), 0.001 (tdc-1 ( $n=129$ animals) vs. tdc-1+OA ( $n=103$ animals)), 0.002 (tdc-1 vs. tdc$1+\operatorname{NE}\left(n=86\right.$ animals)) ( $\left.{ }^{\star} p<0.05,{ }^{\star \star} p<0.005,{ }^{\star \star \star} p<0.001\right)$. e A ser-3 mutant shows Emo. Transgenic lines (ser-3 TG, $n=14$ gonads) rescue ser-3 mutants ( $n=31$ gonads). $\mathbf{f}, \mathbf{g}$ The reproduction periods $(\mathbf{f})$ and the total brood sizes calculated from $\mathbf{f}$ ( $\mathbf{g}$ ) of tbh- 1 ( $n=8$ animals, two-sided $t$-test: $p=0.059), t d c-1$ ( $n=10$ animals, $p=0.003)$ and ser-3 mutants ( $n=9$ animals, $p=0.623$ ). $p$-value is vs. wild type. The mutants and wild-type ( $n=8$ animals) stop reproducing after 3 days as adults. Boxplots show the median, mean (X), interquartile range (IQR). The upper whisker: the maxima smaller than 1.5 times IQR plus the third quartile, the lower whisker: the minima larger than 1.5 times IQR minus the first quartile. $\mathbf{b}$, $\mathbf{c}$, e Scale bar $=20 \mu$ m. Source data are provided as a Source Data file.

collectively enclosed by the contractile peritoneal sheath that surrounds each ovary. Both sheaths surround egg chambers in a mesh-like fashion so that all egg chambers are accessible to OA in the hemolymph ${ }^{31}$. A GAL4 line that drives GFP expression by a $t d c 2$ promoter showed that $t d c 2$ is expressed in all processes and many varicosities or boutons of neurons covering the ovary (Fig. 1c) ${ }^{24}$.

In $D$. rerio, the ovary is composed of ovigerous lamellae ${ }^{12}$, each of which contains developing follicles where oocytes of different developmental stages reside. The GFP expression driven by a $d b h$ promoter shows that the ovary is innervated by norepinephrinergic neurons (Fig. 1d). The nerves run along the main vasculatures of the dorsal side of the ovary. In the cortical areas, the nerves project extensively across the entire lamella, seemingly independent of the vasculature (Fig. 1e, insets) ${ }^{32}$. The processes labeled with GFP consist of many small-size varicosities or boutons from which NE would be released to the ovary, although we did not observe direct innervation of the follicles by $d b h$ expressing neurons. Taken together, these observations suggest that $\mathrm{NE}$ is synthesized and released into the zebrafish ovary.

Maintenance of quiescent oocytes by OA in C. elegans. Due to lack of the second meiotic arrest, when kept unfertilized for long periods of time, a small fraction of $C$. elegans oocytes awaken, spontaneously undergo several rounds of abnormal haploidic mitotic replications and become polyploid endomitotic oocytes. This is called the Endomitotic Oocytes (Emo) phenotype ${ }^{33}$ (Fig. 2a). While the first oocyte (red) is exiting quiescence and resumes meiosis, the remaining oocytes remain quiescent. If the arrest signal is absent and/or the most proximal oocyte blocks the gonad-spermatheca passage, however, the rest of the oocytes become Emo.

After $\sim 90 \mathrm{~h}$ from hatching, sperm are depleted and $25 \%$ of wild-type C. elegans exhibit Emo, whereas $80 \%$ of the $t b h-1$ mutants exhibit Emo (Fig. 2b, d). The tbh-1 mutants carry a deletion covering the $5^{\text {th }}$ and $6^{\text {th }}$ exons and do not produce $\mathrm{OA}^{25}$. In the presence of sperm, the overall reproduction processes of oocyte maturation (examined by MAPK activation), ovulation, and fertilization of $t b h-1$ and $t d c-1$ mutants are indistinguishable from those of wild-type, showing that $\mathrm{OA}$ is not required for oocyte maturation or any process beyond (Fig. 2c and Supplementary videos 1-3). Also, the mutants did not deplete their sperm any sooner than wild-type animals, as the mutants and wild-type stop producing progeny after 3 days as adults. This shows the mutants' reproduction span is similar to that of wild type (Fig. $2 \mathrm{f}, \mathrm{g}$ ), although the $t d c-1$ mutation reduces brood size 
slightly. The Emo phenotype is rescued by adding OA $(20 \mathrm{mM})$ exogenously to the mutants (Fig. 2b, d), indicating that OA is required for maintaining oocyte quiescence and that the Emo phenotype is the result of failure to maintain quiescence. Interestingly, when we treated $t d c-1$ or $t b h-1$ mutants with $\mathrm{NE}$ $(5 \mathrm{mM})$, it partially rescued the Emo phenotype of the mutants, showing that NE could at least partially replace OA function in oocyte quiescence in C. elegans (Fig. 2d).

To identify the $\mathrm{OA}$ receptor for oocyte quiescence, we performed RNAi of all five reported OA receptors, octr-1, ser-3, ser-6, tyra-2, and tyra- $3^{34}$ and found that only ser-3 RNAi produced the Emo phenotype. The ser-3 mutants phenocopied the RNAi result, confirming that SER-3 is the OA receptor (Fig. 2e). It has been suggested that the quiescence signals are produced in gonadal sheath cells that surround oocytes and are then transported to oocytes through gap junctions ${ }^{35}$. SER-3 expression is limited to head muscles, a few neurons, intestine, spermatheca, and gonadal sheath cells ${ }^{36}$. To identify the SER-3 site of action, we targeted ser-3 expression to gonadal sheath cells using a ceh-18 promoter. CEH-18 is a Pit-1/Oct-1,2/Unc-86 (POU) domain-containing transcription factor required for gonadal sheath cell differentiation ${ }^{37}$. Although $\mathrm{CEH}-18$ is broadly expressed in muscles, neurons, and the gonads ${ }^{38}$, within the gonad it is only expressed in gonadal sheath cells and not detected in sperm or oocytes ${ }^{39}$. In addition, the only tissues where both ceh-18 and ser-3 are expressed are gonadal sheath cells and spermatheca. This construct rescues the Emo phenotype in ser-3 mutants (Fig. 2e, ser-3 TG), suggesting that SER-3 function in gonadal sheath cells is sufficient to maintain quiescent oocytes.

\section{Maintenance of quiescent oocytes by $\mathrm{OA}$ in female nematodes.} Next, we tested whether lack of OA results in the Emo phenotype in feminized mutants of C. elegans and in females of a related species, $C$. remanei. When we knocked down $t d c-1$ expression by RNAi in two feminized mutants of C. elegans, fem-1 and fog$2^{40,41}$, the mutants failed to maintain oocyte quiescence and the oocytes became Emo beginning at $24 \mathrm{~h}$ after the $4^{\text {th }}$ larval stage (L4), the last larval stage before the animal becomes an adult (Fig. 3a). When we knocked down $t d c-1$ gene expression by RNAi in C. remanei, a gonochoric (i.e., having males and females) species, the oocytes of the virgin became Emo beginning $24 \mathrm{~h}$ after the L4 stage (Fig. 3b). Together, our results suggest that OA serves as an oocyte quiescence signal in C. elegans and C. remanei.

Oocyte maturation is initiated by sperm signal Major Sperm Proteins (MSPs) and mediated via GSA-1, a canonical $\mathrm{G}_{\alpha \mathrm{s}}$ that activates an adenylyl cyclase to produce cAMP and activate protein kinase A (PKA) in gonadal sheath cells ${ }^{35,42}$. Any mutations in this process block the oocyte maturation and delay ovulation even in the presence of sperm. The oocytes of OA mutants mature normally when the sperm is present, indicating the oocyte maturation process triggered by sperm signal is intact. Yet, because OA maintains oocyte quiescence when sperm is absent, once sperm is present, sperm signal would override OA signaling. To examine the genetic interaction between $\mathrm{OA}$ and sperm signaling, we first generated fog-2; tbh-1 double mutants, which were maintained in the presence of males. Consistent with the RNAi results, fog-2; $t b h-1$ double mutants failed to maintain quiescent oocytes from as young as $10 \mathrm{~h}$ after L4, and the oocytes became round instead of remaining cylinder-shaped as in fog-2 single mutants (Fig. 3c). fog-2; tbh-1 double mutants laid oocytes at a higher ovulation rate than fog- 2 single mutants (Fig. $3 \mathrm{~d}$ ) and became Emo at $1 \mathrm{~d}(19 \%$ Emo, $n=31), 2 \mathrm{~d}(50 \%$ Emo, $n=24)$, and $3 \mathrm{~d}(67 \%$ Emo, $n=24)$ after L4. In contrast, fog-2 females maintain quiescent oocytes and produce no Emo for 3 days $(0 \%$ Emo, $n=35$ ). Exogenous OA rescued fog-2; tbh-1 both for ovulation rate (albeit in a delayed manner, likely because pharmacological action of exogenous $\mathrm{OA}$ action takes time) and Emo as quiescent oocytes accumulated (Fig. 3c, d) (0\% Emo, $n=24)$.

Next, we examined the genetic interaction between $\mathrm{OA}$ and sperm signaling by comparing the ovulation rates between fog-2; tbh-1 and fog-2 under the condition of reduced sperm signaling of $\mathrm{G}_{\alpha \mathrm{s}}$, which is encoded by $g s a-1$. We reasoned that if OA directly interacts with sperm signaling, the gsa-1 phenotype would be epistatic to that of $t b h-1$ in maintaining quiescent oocytes; the phenotype of $g s a-1 \mathrm{RNAi}$ would be the same as that of $g s a-1 \mathrm{RNAi}$ in the tbh-1 background. As $\mathrm{G}_{\alpha \mathrm{s}}$ is also required for spermatheca contraction $^{43}$, the oocytes of $g s a-1$ RNAi-treated fog-2; tbh-1 animals became trapped in the spermatheca, where they matured and became Emo in the gonadal sheath, whereas the oocytes of fog-2 single mutant did not (Fig. 4a, b). Although it is unclear why oocytes of fog- 2 mutants were able to pass spermatheca in $g s a-1$ RNAi treatment, the fact that $g s a-1$ RNAi produces different phenotypes between fog-2 and fog-2; tbh-1 indicates $g s a-1$ is not epistatic to $t b h-1$.

As gsa-1 RNAi-treated fog-2; tbh-1 mutants did not lay unfertilized oocytes, we could not accurately determine the ovulation rate. Instead, we monitored the entire progress of ovulation of a single live animal. Approximately $36 \mathrm{~h}$ after the L4 stage ( $t=0$ in Fig. $4 a, b), 100 \%$ of $g s a-1$ RNAi-treated fog- 2 mutants contained 12 or more cylinder-shaped quiescent oocytes in each gonad arm. In contrast, only $17.7 \%$ of $g s a-1$ RNAi-treated fog-2; tbh-1 mutants contained stacked oocytes and $82.3 \%$ showed no stacked quiescent oocytes $(n=96)$. In addition, the stacked oocytes of gsa-1 RNAi-treated fog-2; tbh-1 mutants are different from normal quiescent oocytes. They are bigger and rounder than those of $g s a-1$ RNAi-treated fog-2 mutants (Fig. $4 \mathrm{~b}, t=0$ ). For two days, gsa-1 RNAi-treated fog-2 mutants maintained a similar number of quiescent oocytes, whereas the stacked oocytes of $g s a-1$ RNAi-treated fog-2; tbh-1 mutants were all exhausted (Fig. $4 \mathrm{~b}$, $t=21)$.

Figure $4 \mathrm{a}, \mathrm{b}$ show the meiotic maturation and ovulation processes of a $g s a-1$ RNAi-treated fog-2 mutant and a $g s a-1$ RNAi-treated fog-2; tbh-1 mutant. As shown in Fig. 4b, at $t=0$, this gsa-1 RNAi-treated fog-2; tbh-1 mutant contains 13 stacked oocytes. The first two oocytes (red arrows) are dark in color and round with no nuclear membrane, indicating they undergo maturation. Twelve hours later, 11 out of 13 stacked oocytes became mature or became Emo (orange arrow indicates the 11th mature oocyte). Twenty-one hours later, all 13 stacked oocytes became Emo. When we evaluated the maturation rate by counting Emo in a given duration as an indirect measurement of an ovulation rate, the rate of gsa-1 RNAi-treated fog-2; tbh-1 $(0.53 \pm 0.20, n=14)$ is faster than that of $g s a-1$ RNAi-treated fog$2(0.083 \pm 0.043, n=74)$, again showing $g s a-1$ is not epistatic to $t b h-1$. Therefore, our data suggest that the OA signaling required for maintaining quiescent oocytes functions independently of the $\mathrm{G}_{\alpha \mathrm{s}}$ signaling that mediates oocyte maturation activated by sperm signal.

We observed that fog-2; tbh-1 double mutants occasionally stacked oocytes in their gonad arms. To determine whether those stacked oocytes are quiescent and intact, we selected 1-d old females that contained stacked oocytes $(42 \%, n=31)$ and mated them with wild-type males. Mated fog-2; tbh-1 mutants produced the Emo phenotype within $5 \mathrm{~h}$ of mating $(76.5 \%$ Emo, $n=17)$, whereas mated fog-2 females or OA-treated fog-2; tbh-1 did not (0\% Emo, $n=13$ ) (Fig. 4c-e). 1-d old fog-2 females rapidly ovulated stacked oocytes upon mating $(17.7 \pm 4.0 \mathrm{~min} / \mathrm{ovulation} /$ arm, $n=13)$ and no quiescent oocytes were left within $5 \mathrm{~h}$ of mating (Fig. 4c). In contrast, when 1-d old adult fog-2; tbh-1 females with stacked oocytes were selected for mating, the stacked 


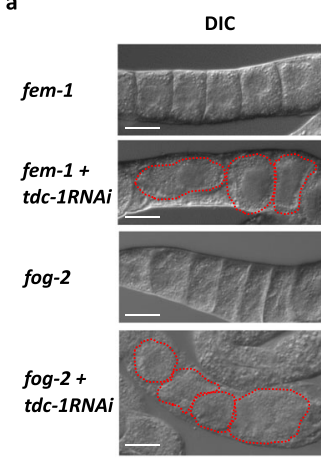

b

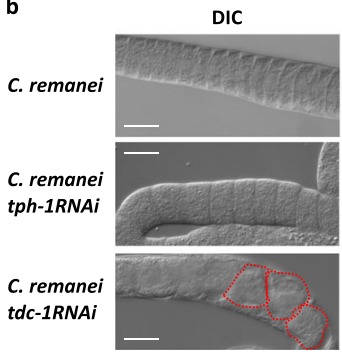

DAPI

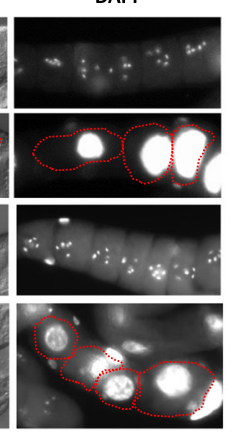

DAPI

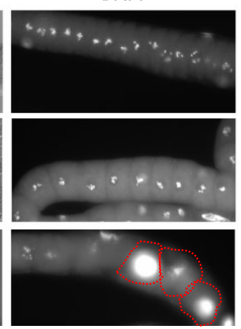

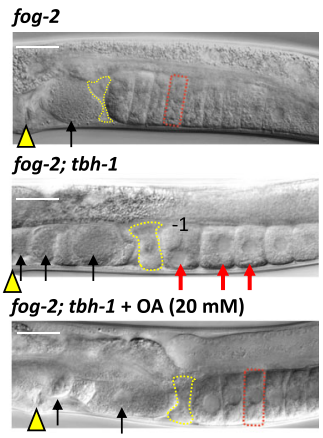

d
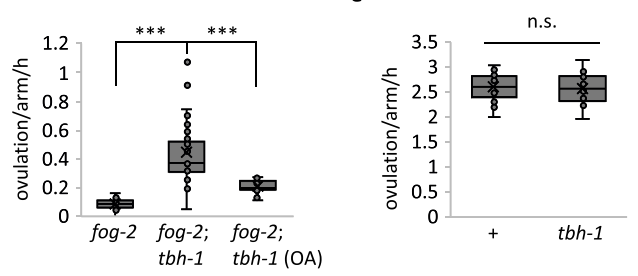

Fig. 3 Maintenance of quiescent oocytes by octopamine in Caenorhabditis females. a fem-1 and fog-2 mutants treated with control or tdc-1 RNAi. Each image represents the 20 - 40 gonad images. Red-dotted circle indicates individual Emo. b A C. remanei female treated with control RNAi or tdc- 1 RNAi. tph1 encodes a tryptophan hydroxylase. Similar images were observed from 33 gonads for an empty vector, 36 gonads for tph-1 RNAi and 23 gonads for tdc- 1 RNAi. c In fog-2 mutants, 6 - 7 quiescent oocytes accumulate (red rectangle). Arrowheads: vulva, yellow polygons: spermatheca. In fog-2; tbh-1 mutants, no quiescent oocytes accumulate. Due to a faster ovulation rate than that of fog- 2 mutants, three unfertilized oocytes in the uterus are shown (black arrows). The nucleus of the most proximal oocyte (-1) migrated to its cortex, indicating it is undergoing meiotic maturation ${ }^{23}$. Red arrows: activated oocytes. OA (20 mM) rescues the fog-2; tbh-1 phenotype. Two unfertilized oocytes are shown in the uterus due to delay of rescue (black arrows). All animals were virgins observed $10 \mathrm{~h}$ after late L4. Similar images were observed from 7 gonads for fog-2, 16 gonads for fog-2; tbh-1 and 9 gonads for fog-2; tbh-1 + OA. a-c Scale bar $=20 \mu \mathrm{m}$. d Ovulation rates of fog-2 ( $n=28$ animals), fog-2; tbh-1 ( $n=71$ animals) and fog-2; tbh-1+OA ( $n=35$ animals). Twosided $t$-test: $p=2.6 \times 10^{-15}\left(^{\star \star \star}\right)$ for fog-2 vs. fog-2; tbh-1, $4.5 \times 10^{-10}\left(^{\star \star \star}\right)$ for fog-2; tbh-1 vs. fog-2; tbh-1+ OA. e Wild-type (+) ( $n=20$ animals) and tbh-1 mutants ( $n=14$ animals) show similar ovulation rates in the presence of sperm. ( $\mathrm{n} . \mathrm{s}$.: not significant, two-sided $t$-test: $p=0.76$ ). d, e Boxplots show the median, mean $(X)$, interquartile range (IQR). The upper whisker: the maxima smaller than 1.5 times IQR plus the third quartile, the lower whisker: the minima larger than 1.5 times IQR minus the first quartile. Source data are provided as a Source Data file.

oocytes became Emo within $5 \mathrm{~h}$ of mating $(n=12)$ (Fig. $4 \mathrm{~d}$, red arrows). The Emo phenotype of fog- 2 ; tbh-1 was rescued by $\mathrm{OA}(20 \mathrm{mM})$ restoring a similar rate of ovulation $(18.9 \pm 2.2 \mathrm{~min} /$ ovulation/arm, $n=11)$. Taking the delay in action of exogenously treated OA into account, we examined ovulation $14 \mathrm{~h}$ after the setup of mating.

This result suggests that the stacked oocytes in fog-2; tbh-1 were not quiescent and that the sperm signal that promotes ovulation rate in $f \circ g-2 ; t b h-1$ females allowed us to visualize the defect. Approximately $40 \%$ embryos of the mated fog- 2 ; tbh-1 females were unable to hatch (Fig. $4 \mathrm{~g}$ ), suggesting the ill-timed awakening of oocytes in the absence of $\mathrm{OA}$ contributes to abnormal fertilization and/or development. When we mated fog2; tbh-1 females at late L4 stage, however, they reproduced normally; they did not produce Emo and the most embryos hatched (Fig. 4f, g). This indicates the oocytes of fog-2; tbh-1 are fully functional as long as they do not need to enter quiescence. When we mated the $t d c-1$ RNAi-treated $C$. remanei females at late L4 stage, they did not produce Emo, either. These results indicate that once oocytes fail to maintain quiescence, introducing sperm afterwards lowers the chance to produce viable progeny. These results support that for these Caenorhabditis species, OA functions as a safeguard to maintain quiescent oocytes and is critical before the sperm signal becomes available.

Maintenance of quiescent oocytes by $\mathrm{OA}$ in $\mathrm{D}$. melanogaster. To test whether OA serves as a conserved signal for oocyte quiescence, we examined D. melanogaster. In D. melanogaster, previtellogenic egg chambers consist of an oocyte and 15 nurse cells, surrounded by a layer of somatic follicle cells ${ }^{44}$. During winter, fruit flies in the wild enter reproductive diapause or dormancy and do not contain egg chambers past stage $8^{14,15,21,22}$. This diapause is considered different from reduced oogenesis by starvation, during which the proliferation rate of follicle cells reduces to $1 / 4$ of that of well-fed condition $14,45,46$. Starved previtellogenic egg chambers share certain defined characteristics of oocyte quiescence in other animal germlines such as redistribution of ribonucleoprotein complex components and cortically condensed microtubules ${ }^{46,47}$. Based on this similarity, hereafter we call reduction of oogenesis or temporary developmental arrest of previtellogenic egg chambers induced by starvation as oocyte quiescence.

We examined OA function in maintaining oocyte quiescence under protein starvation by removing yeast components from the media. Without yeast, the major source of protein ${ }^{48}$, the synthesis of yolk proteins is reduced and the egg chambers grow very slowly ${ }^{45}$. This quiescence of egg chambers is rapidly reversed by a protein-containing diet (Fig. 5a) ${ }^{47,49}$.We examined the ovaries of virgins from immediately after they eclosed, in wild-type (Canton-S or $w^{1118}$ ), $t b h^{n M 18}$ (hereafter called $t b h^{-/-}$) (a null allele that does not produce OA) ${ }^{50}$, and $t b h^{+/ n M 18}$ flies (hereafter called $t b h^{+/-}$) at 0 -day post-eclosion ( $\left.d p e\right), 1 d p e, 1.5 d p e$, and 2 $d p e$ upon protein starvation ( $1 \mathrm{dpe}$ is $24 \mathrm{~h}-, 1.5 \mathrm{dpe}$ is $36 \mathrm{~h}-, 2 \mathrm{dpe}$ is $48 \mathrm{~h}$-post-eclosion). We picked those time points because flies do not lay eggs until $2 \mathrm{dpe}$. This is necessary to examine the sole 


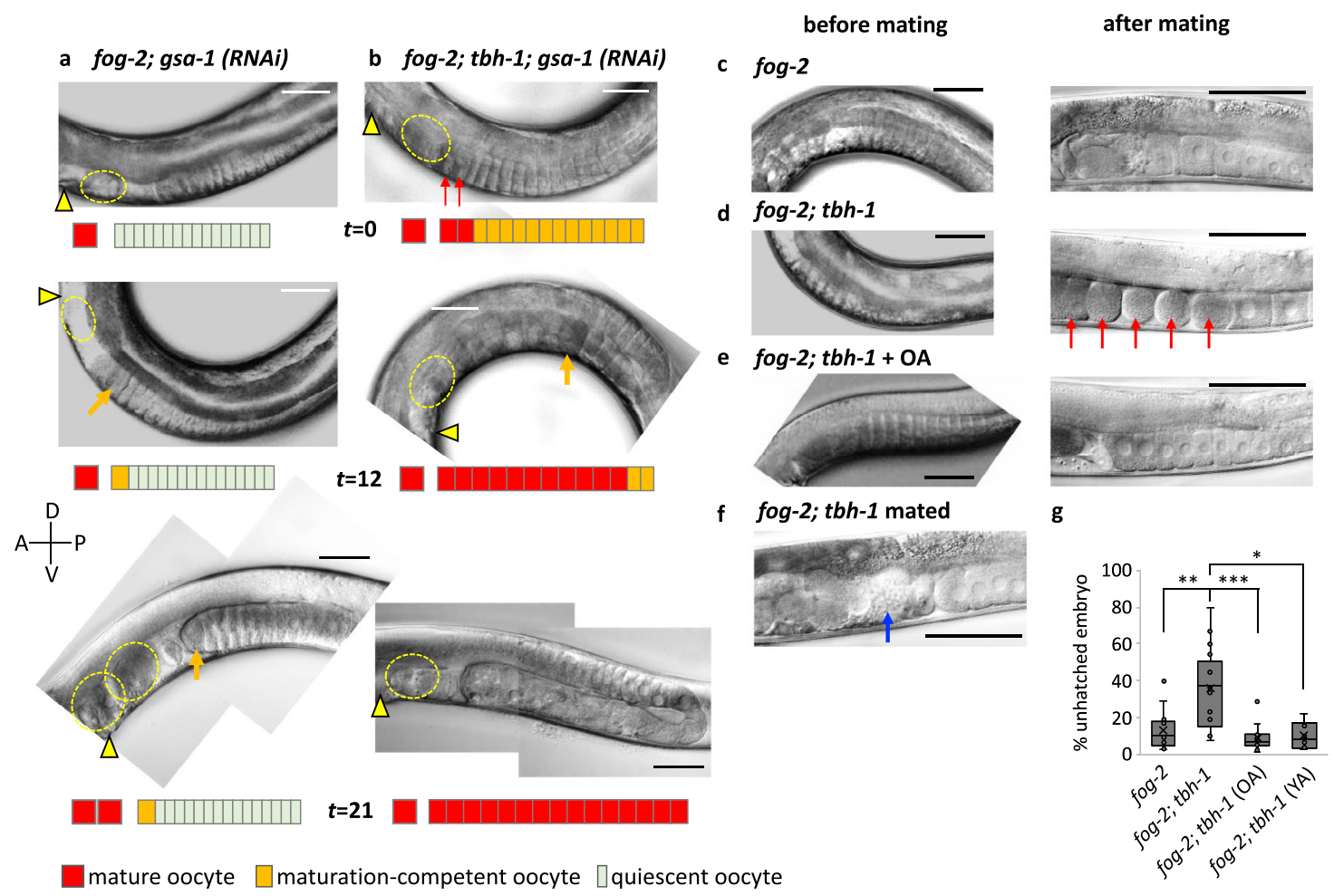

Fig. 4 Octopamine acts in parallel to sperm signaling. a, $\mathbf{b} O A$ negatively regulates ovulation in the absence of sperm signal independent of $\mathrm{G}_{\alpha \mathrm{s}}$ signaling. The ovulation process of a virgin of fog-2 with gsa-1 RNAi (a) or fog-2; tbh-1 with gsa-1 RNAi (b) was observed at $36 \mathrm{~h}(t=0)$, 48 h $(t=12)$, and $57 \mathrm{~h}$ $(t=21)$ after the late L4 stage. Triangle indicates vulva, red arrows mature oocytes, and yellow dotted circle a matured oocyte ovulated in the uterus or trapped inside the spermatheca. At time point 0 , oocytes were stacked in both fog-2; gsa-1RNAi and fog-2; tbh-1; gsa-1RNAi ( $n=12$ gonads for both). c-e Representative images of fog-2 and fog-2; tbh-1 before and after mating ( $5 \mathrm{~h}$ ) and OA treated. Similar images were observed from 13 animals for fog-2, 12 animals for fog-2; tbh-1 and 11 animals for fog-2; tbh-1 + OA. Red arrow: Emo. f Mating does not produce Emo when late L4 females of fog-2; tbh-1 mutants (similar images were observed from 6 animals) were mated with wild-type males. We observed 98 ovulations occurring in $25 \mathrm{~h}(\sim 30 \mathrm{~min} /$ ovulation/arm). Sperm are indicated by the blue arrow). a-f Scale bar $=50 \mu \mathrm{m}$. g Percent unhatched embryos. fog-2 ( $n=400$ embryos), fog-2; tbh-1 $(n=358$ embryos), fog-2; tbh-1 (OA) ( $n=948$ embryos) and fog-2; tbh-1 (YA) ( $n=437$ embryos). Two-sided $t$-test: $p=0.0012$ (**) for fog-2 vs. fog-2; tbh-1, $p=0.0001$ ( $\left.^{\star \star \star}\right)$ for fog-2; tbh-1 vs. fog-2; tbh-1 + OA, $p=0.28$ for fog-2 vs. fog-2; tbh-1+OA. Boxplots show the median, mean (X), interquartile range (IQR). The upper whisker: the maxima smaller than 1.5 times IQR plus the third quartile, the lower whisker: the minima larger than 1.5 times IQR minus the first quartile. Source data are provided as a Source Data file.

effect of OA on oogenesis because $t b h^{-/-}$mutants are defective in egg-laying ${ }^{24}$ and accumulation of full-grown stage 14 egg chambers would interfere with the analysis of the phenotypes if we examine the oogenesis after $2 d p e$. At $0 d p e$, immediately after eclosion, most of the ovaries of all flies contained stage 6/7 egg chambers. The size and the number of total egg chambers of $t b h^{-1-}$ flies were similar to those of $w^{1118}$ flies, although due to a background difference, Canton-S contains fewer egg chambers (Fig. 5b). At $1 d p e$, however, most ovaries of $t b h^{-1-}$ contained further grown egg chambers, such as stage $10 \mathrm{~B}$ or 12 egg chambers, whereas most $t b h^{+/-}$ovaries contained mainly smallsized stage 8 egg chambers (Fig. $5 c$, d). At 1.5 dpe and 2 dpe, most $t b h^{-1}$ ovaries contained many full-grown stage 14 egg chambers, whereas a few $t b h^{+/-}$ovaries contained stage 14 egg chambers (Fig. 5c, e, f). This result suggests that egg chambers stop growing upon starvation in an OA-dependent manner and that accumulation of stage 14 egg chambers in $t b h^{-/-}$mutants is due to failure to maintain quiescent egg chambers.

In D. melanogaster, there are four G-protein-coupled OA receptors. OAMB (OctopAmine receptor in Mushroom Bodies) is similar to vertebrate $\alpha$-adrenergic receptors and essential for egglaying $^{30}$, whereas the other three $(\mathrm{OA} 2 / \operatorname{Oct} \beta 1 \mathrm{R}, \operatorname{Oct} \beta 2 \mathrm{R}, \operatorname{Oct} \beta 3 \mathrm{R})$ are similar to $\beta$-adrenergic receptors ${ }^{51}$. Among the three $\beta$ adrenergic receptors, we tested a mutant of oct $\beta 2 R$, oct $\beta 2 R f 05679$. $A n$ in situ hybridization study showed oct $\beta 2 R$ expression in the nurse cells of previtellogenic egg chambers ${ }^{52}$, suggesting it might be a receptor for the OA action in oocyte quiescence. The allele oct $\beta 2 R^{f 05679}$ contains a piggyBac insertion and is considered to be a significantly reduced-function mutant ${ }^{53}$. The mutant shows a similar phenotype to $t b h^{-/-}$; at $2 d p e$ upon starvation, their ovaries contained mainly stage 14 egg chambers (Fig. 6a, b). Exogenous OA $(5 \mathrm{mg} / \mathrm{ml})$ rescued the unrestrained growth of egg chambers in the $t b h^{-/-}$mutant upon starvation, confirming that lack of OA caused the defect (Fig. 6c) ${ }^{50}$. Exogenous OA could not rescue the defect of oct $\beta 2 R^{f 05679}$ (oct $\beta 2 R^{-/-}$) mutants, however, supporting the idea that $\operatorname{Oct} \beta 2 \mathrm{R}$ is a receptor for OA because without the receptor, adding the ligand will not rescue the phenotype. Together, our data suggest OA is a signal to maintain oocyte quiescence during protein starvation in D. melanogaster.

Next, we asked how OA signaling balances with nutrient signaling and whether OA can inhibit oocyte growth competing with nutrient signals when, for instance, the nutrients are insufficient to support rapid oogenesis. To address this, we depleted nutrients completely and then counted the number of stage 14 egg chambers at $2 d p e$ varying nutrient levels. Under the no nutrient condition, control virgins produce no or few stage 14 egg chambers, whereas $t b h^{-/-}$virgins produce stage 14 egg chambers at a similar rate to that of Canton-S virgins fed on CSY standard media (Fig. 6d. Compare $t b h^{-1-}$ in Fig. $6 \mathrm{~d}$ to $2.5 \%$ in Fig. $6 \mathrm{f}) . t b h^{+/-}$virgin flies produce numbers of stage 14 egg 
a

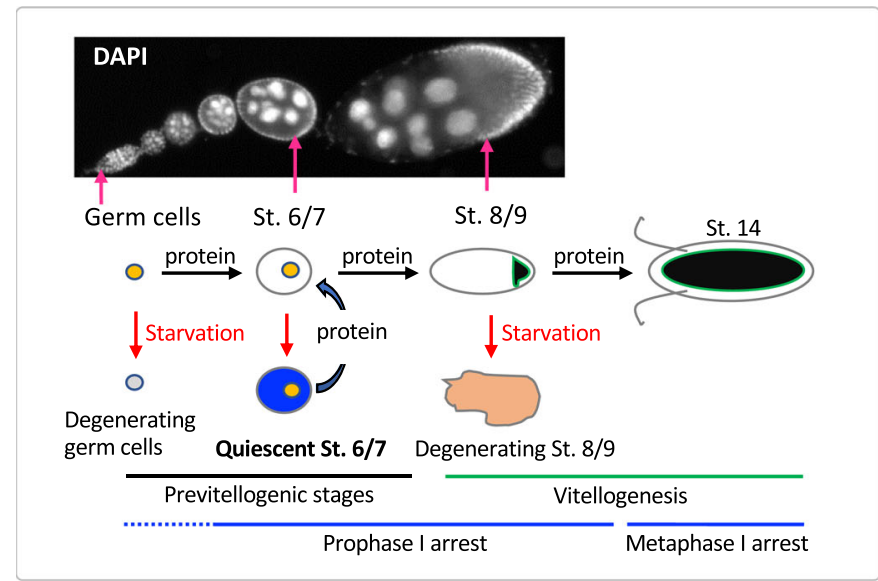

b

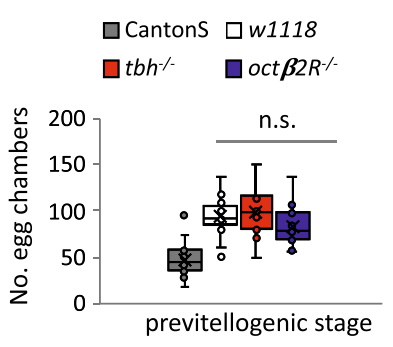

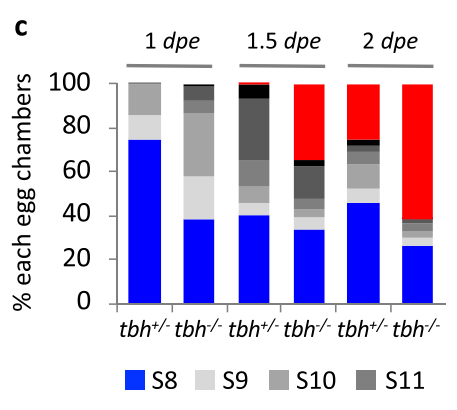
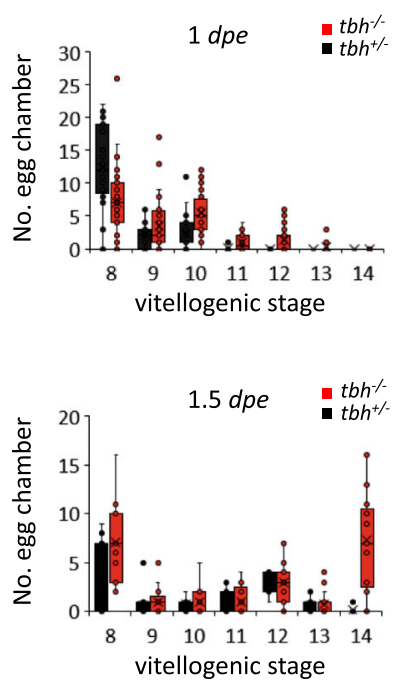

f

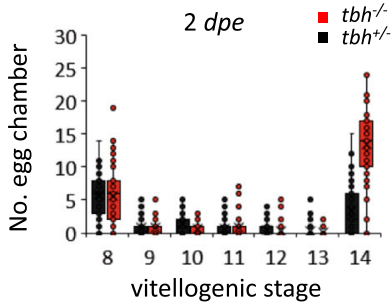

Fig. 5 Octopamine is required for maintaining previtellogenic egg chambers upon nutrient deprivation in D. melanogaster. a Oogenesis of D. melanogaster. Upon protein starvation, either germ cells or stage 8/9 egg chambers are degenerated. The previtellogenic egg chambers slow down the growth, then resume the growth within $2 \mathrm{~h}$ once protein is available. During egg chamber growth, meiotic chromosomes arrest at the prophase I until stage 13 (blue lines). b The number of previtellogenic egg chambers (stages $2-7$ ) per fly are similar between wild-type ( $w^{1118}$ ) female virgin controls $(n=13$ animals $)$ and the octopamine mutant female virgins $\left(t b h^{-/-}(n=11\right.$ animals $)$ and oct $\beta 2 R^{-/-}(n=14$ animals $\left.)\right)$. Two-sided $t$-test: $p=0.63$ ( $w^{1118}$ vs. $\left.t b h^{-/-}\right), 0.22\left(w^{1118}\right.$ vs. oct $\left.\beta 2 R^{-/-}\right), 0.12\left(t b h^{-/-}\right.$vs. oct $\left.\beta 2 R^{-/-}\right)$. Canton-S $(n=35)$ has fewer number of egg chambers than $w^{1118} . p=5.5 \times 10^{-11}$ (Canton-S vs. $w^{1118}$ ), $1.7 \times 10^{-10}$ (Canton-S vs. $t b h^{-/-}$), $1.8 \times 10^{-8}$ (Canton-S vs. oct $\beta 2 R^{-/-}$). n.s.: not significant. c The percent of each stage of egg chambers in the ovaries of $t b h^{+/-}$and $t b h^{-/-}$virgins during the protein starvation period. The percent of stage 14 egg chambers increases in tbh-/mutants compared to that of the heterozygote control, $t b h^{+/-}$. These data are extracted from the raw data shown in $\mathbf{d}-\mathbf{f}$. $\mathbf{d}-\mathbf{f}$ The distribution of each stage egg chambers in the ovaries of each tbh ${ }^{+/-}(n=29$ animals for $1 \mathrm{dpe}, 14$ animals for $1.5 \mathrm{dpe}, 59$ animals for $2 \mathrm{dpe})$ and tbh $h^{-/-}(n=32$ animals for $1 \mathrm{dpe} ; 17$ animals for $1.5 \mathrm{dpe}, 59$ animals for $2 \mathrm{dpe}$ ) female virgin during the protein starvation period. At each time point, tbh ${ }^{-/-}$mutants contain more progressed egg chambers compared to $t b h^{+/-} . \mathbf{b}, \mathbf{d}-\mathbf{f}$ Boxplots show the median, mean $(\mathrm{X})$, interquartile range (IQR). The upper whisker: the maxima smaller than 1.5 times IQR plus the third quartile, the lower whisker: the minima larger than 1.5 times IQR minus the first quartile. Source data are provided as a Source Data file.

chambers that range between wild-type and $t b h^{-1-}$, suggesting haploinsufficiency of OA (Fig. 6d). Exogenous OA restores oocyte arrest to $t b h^{+/-}$and $t b h^{-1-}$ mutants in a concentration dependent manner (Fig. 6e). When we varied the level of nutrients using $2.5 \%$ yeast (CSY2.5, rich media) or $1 \%$ yeast (CYS1\%, poor media), flies on CSY2.5\% produced more stage 14 egg chambers than those on CSY1\% (Fig. 6f), indicating that the number of stage 14 egg chambers increases as nutrients increase. Exogenous OA $(5 \mathrm{mg} / \mathrm{ml})$ inhibits the production of stage 14 egg chambers on poor media but not on rich media, suggesting rich nutrients override the inhibitory signal of OA (Fig. 6f). However, even on rich media, the production of stage 14 egg chambers is reduced at a higher concentration of $\mathrm{OA}(10 \mathrm{mg} / \mathrm{ml})$ (Fig. $6 \mathrm{f}$ : $2.5 \%+\mathrm{OA} 10)$. These results demonstrate the role of OA in balancing nutrient signaling to maintain quiescent egg chambers.

Maintenance of quiescent oocytes by NE in $D$. rerio. Next, we asked whether noradrenergic signal in a vertebrate also plays a role in maintaining quiescent oocytes by examining zebrafish ovaries after prolonged starvation. The five stages of oocytes are easily distinguishable based on size and shape ${ }^{12}$; the stage I oocytes are small, transparent $(<0.14 \mathrm{~mm}$ in diameter $)$, and encapsulated by a layer of pre-granulosa cells forming primordial follicles. At the beginning of stage II, protein- and carbohydratecontaining small cortical alveoli start to accumulate, which occupy much of the ooplasm as the oocyte reaches the later stage II with a diameter of $0.27-0.34 \mathrm{~mm}$ (Fig. 7a). Stage III is the major growing stage where the oocytes become opaque due to vitellogenesis and the diameter of an oocyte reaches up to $0.69 \mathrm{~mm}$. The oocytes mature through stage IV and become eggs (stage V) with a diameter around $0.75 \mathrm{~mm}^{12}$.

Zebrafish contains a single $d b h$ gene to convert dopamine to NE (Fig. 1a). To investigate the NE function in oocyte quiescence, we examined a $d b h^{-1-}$ allele that contains a four nucleotide insertion, which causes a premature stop codon and produces non-functional enzymes ${ }^{26}$. When we compared oogenesis between $d b h^{-1-}$ and $d b h^{+/-}$females after 24 days of starvation, $d b h^{+/}$ovaries contained mostly primordial follicles of stage I, a small fraction (about 5\% of the total follicles) of activated previtellogenic follicles of late stage II and several mature oocytes (stage IV). However, most ovaries did not contain vitellogenic 


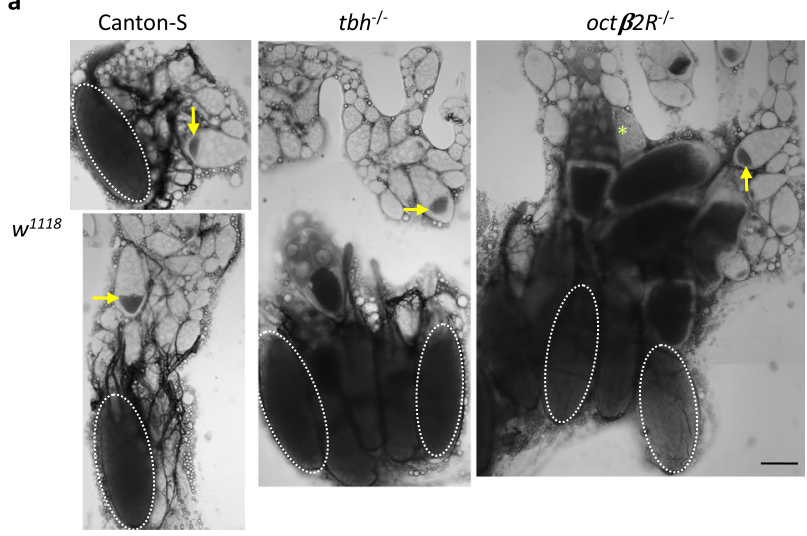

b
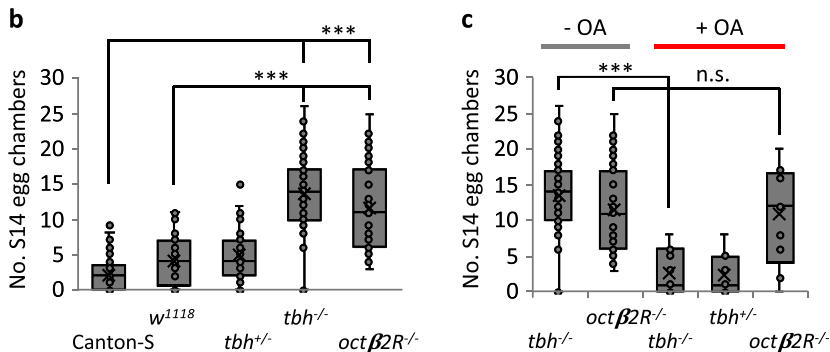
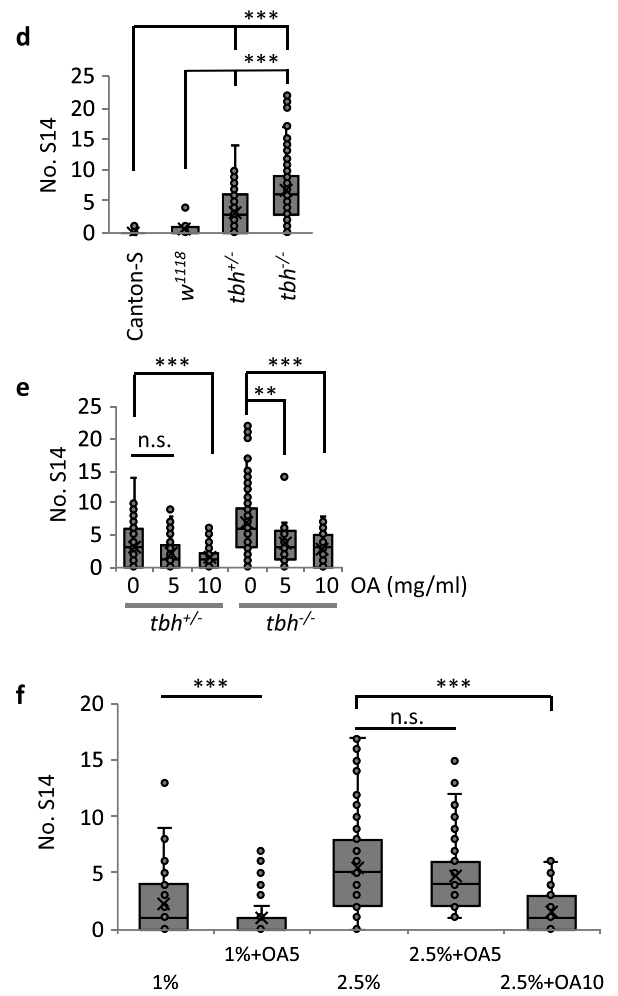

Fig. 6 Octopamine regulates quiescence via Oct $\beta 2 \mathbf{R}$, competing with nutrients. $\mathbf{a}$, b Upon protein starvation, tbh $h^{-/-}$or oct $\beta 2 R^{-/}-$virgins contain more stage 14 egg chambers (S14, white dotted ellipses) than controls. Representative images (Scale bar $=100 \mu \mathrm{m})$ (a) and the quantification (b). Arrows indicate stage 9. $n=85 \mathrm{tbh}^{-/-} ; 35$ oct $\beta 2 \mathrm{R}^{-/-} ; 137$ Canton-S; $52 w^{1118 ;} ; 4 \mathrm{tbh} h^{+/-}$. Two-sided $t$-test: $p=$ CS vs. $w^{1118}(0.0001) ;$ vs. tbh $h^{+/-}\left(1.2 \times 10^{-6}\right) ;$ vs. tbh ${ }^{-/-}\left(8.4 \times 10^{-36}\right)$; vs. oct $\beta 2 R^{-/-}\left(1.4 \times 10^{-10}\right)$, w $w^{1118}$ vs. $t b h^{+/-}(0.21)$; vs. tbh $h^{-/}\left(2.6 \times 10^{-25}\right)$; vs. oct $\beta 2 R^{-/-}\left(2.3 \times 10^{-10}\right) ; 7.2 \times 10^{-21}\left(t b h^{-/-}\right.$vs. tbh ${ }^{+/-}$); $8.8 \times 10^{-7}$ ( $\left(b h^{+/-}\right.$vs. oct $\beta 2 R^{-/-}$); 0.070 (tbh $h^{-/-}$vs. oct $\beta 2 R^{-/-}$). c Exogenous OA $(5 \mathrm{mg} / \mathrm{ml})$ rescues tbh ${ }^{-/-}$mutants, but not oct $\beta 2 R^{-/-}$. $n=85$ animals $(-\mathrm{OA}), 11$ animals $(+\mathrm{OA})$ for $t b h^{-/}-; 35$ animals $(-\mathrm{OA}), 9$ animals $(+\mathrm{OA})$ for oct $\beta 2 R^{-/}-; 7$ animals $(+\mathrm{OA})$ for tbh ${ }^{+/-}$. Two-sided $t$-test: $p=7.5 \times 10^{-9}$ for $t_{b h} h^{-},-0.77$ for oct $\beta 2 R^{-/-}$between untreated and treated with OA. The same data for without OA in Fig. $6 \mathrm{~b}$ was replotted. $\mathbf{d}$ Without nutrients, controls produce few S14 $\left(n=25\right.$ Canton-S, $\left.11 w^{1118}\right)$, whereas $t b h^{-/-}(n=99$ animals $)$ produces at a similar rate to that of Canton-S fed on CSY. tbh $h^{+/-}\left(n=116\right.$ animals) produces S14 ranging between wild-type and tbh ${ }^{-/-}$. Two-sided $t$-test: $p=$ CS vs. $w^{1118}(0.05)$, vs. tbh $h^{+/-}\left(3.1 \times 10^{-6}\right)$, vs. tbh $h^{-/-}$ $\left(1.7 \times 10^{-10}\right)$, w $w^{1118}$ vs. $t b h^{+/-}(0.008)$, vs. $t b h^{-/-}\left(4.4 \times 10^{-5}\right), 9.4 \times 10^{-10}\left(t b h^{+/-}\right.$vs. $\left.t b h^{-/-}\right)$. e Exogenous OA restores oocyte quiescence to tbh ${ }^{+/-}$ and $t b h^{-/-}$in a concentration dependent manner. $n=33 \mathrm{tbh}+/-, 28 \mathrm{tbh} h^{-/-}$for $5 \mathrm{mg} / \mathrm{ml} \mathrm{OA}, 43 \mathrm{tbh}+/-, 26 \mathrm{tbh}-/-$ for $10 \mathrm{mg} / \mathrm{ml} \mathrm{OA}$. Two-sided $t$-test:

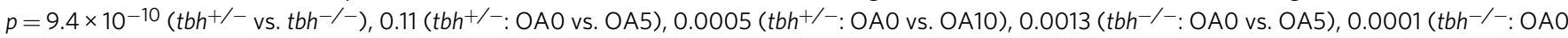
vs. OA10). $\mathbf{f} \mathrm{OA}$ and nutrients compete in regulation of egg chamber growth. $n=55$ Canton-S (CSY1\%), 73 Canton-S (1\% + OA5), 107 Canton-S (CSY2.5\%), 79 Canton-S (CSY2.5\% + OA5), 53 Canton-S (CSY2.5\% + OA10). Two-sided t-test: $p=0.0006$ (CSY1\% vs. CSY1\% + OA5), 0.21 (CSY2.5\% vs. CSY2.5\% + OA5) $1.4 \times 10^{-7}$ (CSY2.5\% vs. CSY2.5\% + OA10). b-f Numbers of egg chambers per fly were shown. Boxplots show the median, mean $(X)$, interquartile range (IQR). The upper whisker: the maxima smaller than 1.5 times IQR plus the third quartile, the lower whisker: the minima larger than 1.5 times IQR minus the first quartile. Source data are provided as a Source Data file.

follicles of stage III (Fig. 7b, d, f, g). This observation suggests that under long-term starvation, most primordial follicles become quiescent in $d b h^{+/-}$ovaries and only a small fraction of total follicles undergo PFA. After 45-day starvation, the $d b h^{+/-}$ovaries contain only stage I follicles (Fig. 7h), suggesting that follicles in other stages eventually disappear and only the stage I oocytes remain quiescent as starvation continues.

In contrast, after 24 days of starvation, $d b h^{-/-}$ovaries contain many late stage II and vitellogenic stage III follicles (Fig. 7c, reddotted circle as examples). $d b h^{-/-}$ovaries contain stage II follicles even after 32 days of starvation (about 18\% of the total follicles, Fig. $7 \mathrm{e}-\mathrm{g}$ ). Most strikingly, every starved $d b h^{-/-}$ovary we examined contained early stage III follicles $(n=22)$, which we rarely observed in $d b h^{+/-}(n=14)$. This suggests that in the absence of $\mathrm{NE}$ the follicles keep growing and reach the early vitellogenic stage, which does not happen in the presence of NE. Taken together, these results suggest that NE is required for maintaining oocyte quiescence in zebrafish.

After three-weeks of starvation, late stage III oocytes $(0.34-0.69 \mathrm{~nm}$ in diameter $)$ are absent in both $d b h^{-1-}$ and $d b h^{+/-}$ovaries (Fig. 7b-e). This suggests that as in flies, if the starvation happens after the follicle has passed stage III, the follicle degenerates due to lack of building blocks and energy. The fact that late stage III oocytes are absent in both $d b h^{-}-$and $d b h^{+/-}$ovaries also indicates that $\mathrm{NE}$ is not required for degeneration of oocytes.

Consistent with a previous report, $d b h^{-/-}$female zebrafish reproduce normally under well-fed conditions ${ }^{26} \cdot d b h^{-1-}$ mutant females produce $100-300$ eggs upon mating, and the ovaries do not show any morphological differences from those of wild-type. Also, starvation-induced quiescence of oocytes is reversible; upon refeeding, the starved fish resumed oogenesis within 2 days.

\section{Discussion}

Maintaining quiescent oocytes is pivotal for quality reproduction span. We identified the noradrenergic signal as a conserved oocyte quiescence signal in two Caenorhabditis species while the oocytes are waiting for sperm, and in D. melanogaster and $D$. rerio when they are deprived of nutrients. The ovaries of all four 

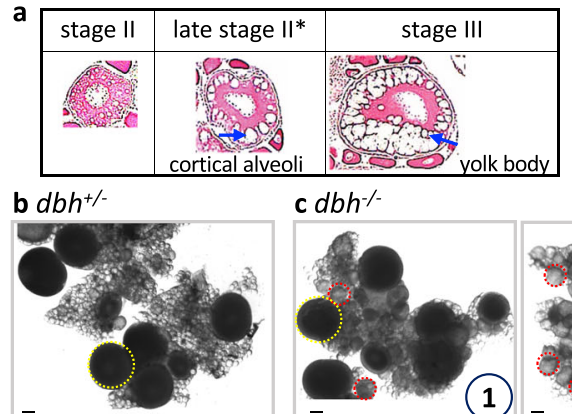

c $d b h^{\%-}$

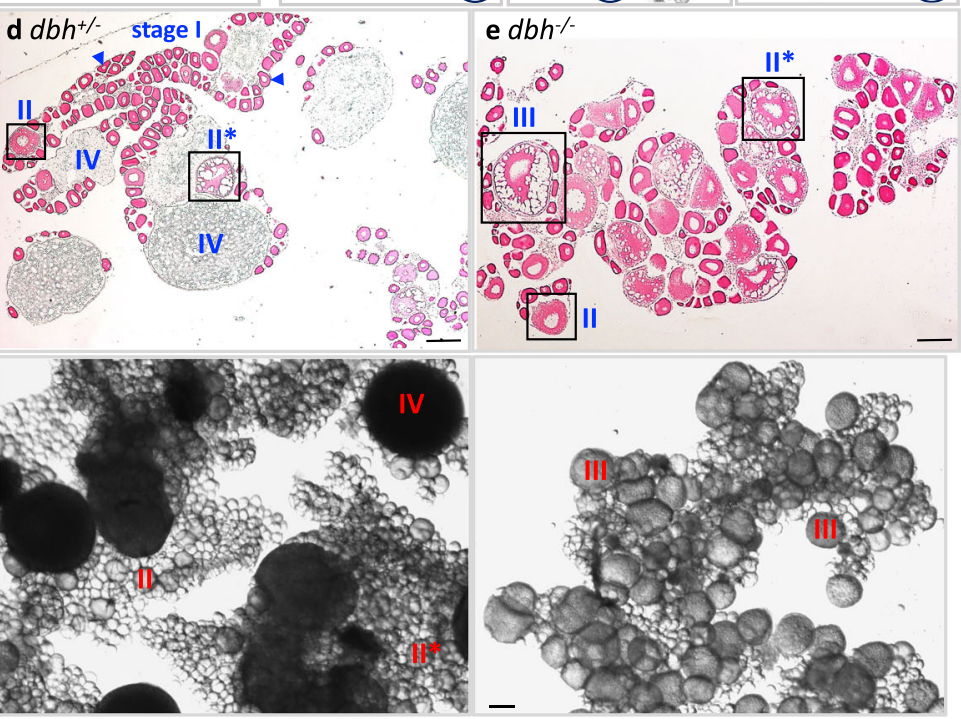

f

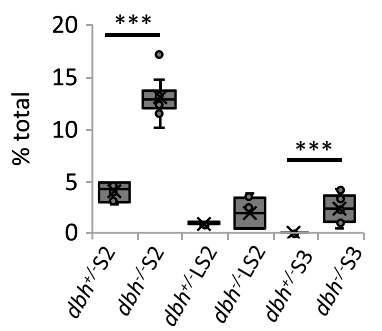

h $45-d d b h^{+/-}$
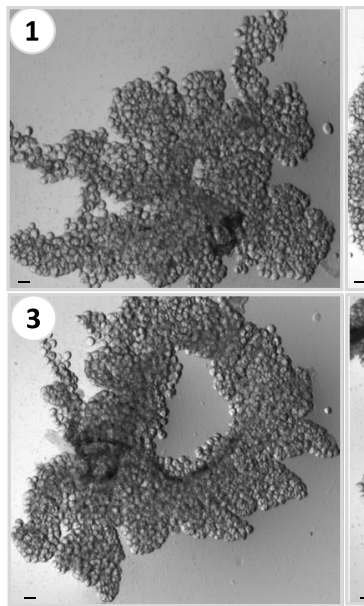

g

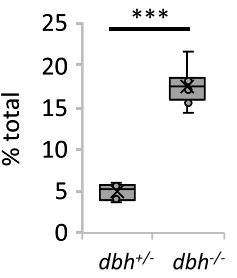

Fig. 7 NE is necessary for maintenance of quiescent oocytes during starvation. a Follicle images of each stage of oocytes based on size and shape. b A representative image of an ovary of 24-d starved $\mathrm{dbh}^{+/-}$- shows two major populations of oocytes of stage IV (dark and big, yellow circle) and stage I (transparent and small). Similar images were observed from 9 samples. c Images of three different ovaries of $24-\mathrm{d}$ starved $d b h^{-/-}$. In contrast to $d b h^{+/-}$, most oocytes are stage II or III (intermediate size, red circle). Similar images were observed from 14 samples. $\mathbf{d}$ Upper panel: a representative image of an ovary of 24-d starved $\mathrm{dbh} \mathrm{h}^{+/-}$stained with neutral red. Stage I oocytes are most abundant (arrowheads). Oocytes in each stage of II, II (late stage II), and IV are easily visible. Lower panel: an image of the whole ovary. e A representative image of an ovary of 32-d starved $d b h^{-/-}$. Upper panel: neutral-red stained image. Compared to $\mathrm{dbh}^{+/-}$, oocytes of later stages (e.g., stages II and III) are abundant. Lower panel: an image of the whole ovary. b-e Scale bar $=200 \mu \mathrm{m}$. f The percent of oocytes at stage II (S2), late stage II (LS2), and stage III (S3) in $d b h^{+/-}$( $n=8$ sections from three independent fish) and $d b h^{-/-}\left(n=10\right.$ sections from four independent fish). The stage III oocytes were only observed in $d b h^{-/-}$. Two-sided $t$-test: $p=3.1 \times 10^{-9}$ between $d b h^{+/-}$ vs. $d b h^{-/-}$in stage II; $p=0.04$ in late stage II; $p=0.0004$ for stage III. $\mathbf{g}$ The sum of stage II, late stage II, and stage III oocytes presented in $\mathbf{f}$. Two-sided t-test: $p=2.3 \times 10^{-11}$. f-g Boxplots show the median, mean $(X)$, interquartile range (IQR). The upper whisker: the maxima smaller than 1.5 times IQR plus the third quartile, the lower whisker: the minima larger than 1.5 times IQR minus the first quartile. $\mathbf{h}$ Ovaries of four different $d b h^{+/-}$females after 45 days of starvation contain only stage I oocytes. Ovaries of four other females showed similar phenotype. Scale bar $=100 \mu \mathrm{m}$. Source data are provided as a Source Data file.

species are heavily innervated by cells producing OA or NE. Failure of oocyte quiescence results in endomitotic oocytes, and then sterility in the two Caenorhabditis species, unrestrained growth of egg chambers in D. melanogaster, and excess activation of primordial follicles in $D$. rerio.

In C. elegans, $\mathrm{OA}$ is essential for maintaining quiescent oocytes when the sperm signal is absent. We observed that in fog-2; tbh-1 mutants, $\mathrm{OA}$ is not required if the sperm signal is present early enough to prevent accumulation of quiescent oocytes; both the tbh-1 mutant hermaphrodite and the fog-2; tbh-1 females mated from L4s reproduce normally without producing Emo phenotypes. C. elegans oocytes are not individually surrounded by somatic follicle cells as in mammals. Instead, they grow inside the gonadal sheath communicating with them via gap junctions, which therefore serves a function similar to the follicle cells in mammals for maintaining quiescent oocytes. In ceh-18 or inx-14/ 22 mutants, which show abnormal sheath differentiation or lack of gap junctions to oocytes, respectively, quiescent oocytes are not maintained, resulting in faster ovulation and frequent Emo in the absence of sperm $37,39,54$. In addition, certain G-protein signals, such as $G_{\alpha o}$, are known to play a role in the gonadal sheath in maintaining oocyte quiescence ${ }^{54}$. As our results suggest that OA signal does not directly interact with $G_{\alpha s}$, which mediates the sperm signal, we suggest that $\mathrm{OA}$ from gonadal sheath cells may act on SER-3, a G-protein-coupled receptor, in an autocrine fashion and produce downstream inhibitory signals which are transported to the oocytes through gap junctions and maintain oocyte quiescence.

Upon nutrient deprivation, D. melanogaster virgins slow down the growth of previtellogenic egg chambers, which remain quiescent until nutrients are available. Our data suggest that $\mathrm{OA}$ is required for maintaining oocyte quiescence. $t b h^{-/-}$mutants could sense D-glucose in gustatory sensory neurons upon starvation and consume the same amount of food as wild-type under both fed and starved conditions, showing that the hunger sensation in the mutant is intact ${ }^{55,56}$. This excludes the possibility that OA may be directly involved in sensing nutrient homeostasis and the mutants fail to evaluate the nutrient condition and, in 
turn, fail to maintain oocyte quiescence. Rather, OA appears to regulate oocyte quiescence directly, as the ovaries have access to $\mathrm{OA}$ from OA-expressing neurons innervating the ovarian epithelium $^{51,57,58}$

Nutrient-sensing pathways play a major role in promoting the growth of egg chambers and follicles; in flies, a high level of dILPs and $\mathrm{JH}$ promote the growth of the egg chamber ${ }^{19,22}$. In mammals, the mTOR and the insulin-FOXO pathways coordinate to awaken quiescent oocytes ${ }^{4}$. The fact that nutrient-sensing pathways regulate the timing of oocyte awakening suggests that deprivation of nutrients would do the opposite and stop oogenesis. Indeed, a reduced level of insulin signal halts the growth of egg chambers in flies ${ }^{22}$. It remains unclear, however, whether the absence of a nutrient signal is sufficient to maintain quiescent oocytes. Our results that the nutrient signal and OA signal could balance each other suggest that when the level of nutrients is insufficient, OA signal could be critical to maintain quiescent egg chambers.

Although our zebrafish study was based on observations made from a mutant and we have not identified the receptor(s) and the action site in the ovary, we could observe that the quiescent oocytes were maintained in the control whereas they were not in the mutant fish, which is the same as we observed in the invertebrate animals that we examined. Based on these results, we suggest that the nutrient-responsive neuronal signals are necessary for promoting and stopping the oogenesis and that $\mathrm{OA} / \mathrm{NE}$ are necessary for maintaining the quiescent oocytes once the nutrient-responsive signals are sufficiently reduced. The ovary receives noradrenergic input in both vertebrates and invertebrates; NE is released from the sympathetic nerves innervating ovary and regulates steroidogenesis and early follicular development in mammals ${ }^{59,60}$. In addition, starvation activates sympathetic nerves ${ }^{61}$ and thus would lead to release of NE into the ovary. OA production in C. elegans is also increased under starvation ${ }^{62}$. These observations may suggest that unfavorable conditions for reproduction such as starvation would increase noradrenergic input in the ovary.

Based on our results, we propose a model of noradrenergic signaling in oocyte quiescence (Fig. 8). In C. elegans, quiescent oocytes are maintained by OA. Sperm override OA's maintenance signal and induce oocyte maturation. Nutrients probably would not play a significant role in controlling oocyte maturation because, unlike other animals, reduced vitellogenesis in C. elegans does not severely hamper embryogenesis ${ }^{63}$. In D. melanogaster and $D$. rerio, quiescent oocytes are maintained by OA/NE in the absence (or at low level) of nutrient signals. Availability of nutrients overrides this maintenance signal, and oocytes grow and mature (Fig. 8a). Our results in Drosophila and zebrafish suggest that the onset of oogenesis should be regulated by a delicate balance between two signaling systems: signals for abundant nutrients mediated by insulin and amino acids and signals for nutrition deprivation by the noradrenergic signal. The role of $\mathrm{OA} / \mathrm{NE}$ would be the most relevant and critical when nutrients are insufficient for continuous oogenesis (Fig. 8b). OA/NE serves as a counterbalance signal to nutrients; increased levels of $\mathrm{OA} / \mathrm{NE}$ causes oogenesis to stop, and oocytes become quiescent waiting for conditions to improve. In the wild, where availability of food is unpredictable, maintaining oocyte quiescence or releasing them from quiescence has to be determined after careful integration of the two signals. It is intriguing to speculate that OA/NE as an inhibitory signal safeguards and reins in oocyte growth so that even if the condition becomes temporarily better, the oocytes do not instantly commit to full throttled growth unless good conditions persist. If our model is correct then, when the ratio of OA/ NE signal to nutrient signals is abnormally low, the imbalance would activate primordial follicles to grow even under unfavorable conditions and lead to exhaustion of the oocyte pool in mammals. Interestingly, it is reported that many polycystic ovarian syndrome (PCOS) patients who show an increase in the number of antral follicles, abnormal ovulation, and occasional sterility have either an excessive amount of NE with lower $\beta$ adrenergic receptor activity ${ }^{64}$ or an undetectable amount of $\mathrm{NE}^{59,64,65}$. Considering many PCOS patients also suffer from diabetes or obesity-related syndromes, it is possible that they have an abnormally low ratio of NE to nutrient signals, which might contribute to development of PCOS.

Due to lack of directly connected blood vessels or sympathetic nerves, primordial follicles in vertebrates receive nutrients and NE by diffusion from stromal blood vessels and nearby neuronal varicosities, respectively ${ }^{66}$. Mice lacking NE are fertile and do not show severe reproductive problems ${ }^{27}$. Our study suggests that the role of NE is hidden under well-fed conditions, where a high level of nutrient signal would override NE's inhibitory signal. Interestingly, calorie-restricted female mice and rats show a significantly lower proportion of the early stage of the growing follicles and a higher proportion of the quiescent primordial follicles than well-fed animals ${ }^{67,68}$. As antral follicles are the major stage that degenerates during starvation, the higher

\begin{tabular}{|c|c|c|}
\hline Quiescent oocyte & Vitellogenesis & Embryogenesis \\
\hline Meiotic arrest I & $O A$ & Emoryogenesis \\
\hline \multicolumn{3}{|l|}{ D. melanogaster } \\
\hline $\begin{array}{l}\text { Quiescent oocyte } \\
\text { Stages } 6 / 7 \\
\text { Meiotic arrest I }\end{array}$ & 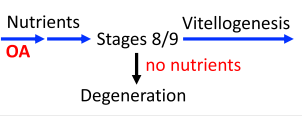 & $\begin{array}{l}\text { Stage } 14 / \text { Egg } \\
\text { Meiotic arrest II }\end{array}$ \\
\hline \multicolumn{3}{|l|}{ D. rerio } \\
\hline $\begin{array}{l}\text { Quiescent oocyte } \\
\text { Stage I } \\
\text { Meiotic arrest I }\end{array}$ & $\underset{\mathrm{NE}}{\stackrel{\text { Nutrients }}{\longrightarrow}} \underset{\substack{\downarrow_{\text {Degeneration }} \text { Stage } \\
\text { noll nutrients }}}{\stackrel{\text { Vitellogenesis }}{\longrightarrow}}$ & $\begin{array}{l}\text { Stage IV/Egg } \\
\text { Meiotic arrest II }\end{array}$ \\
\hline
\end{tabular}

b

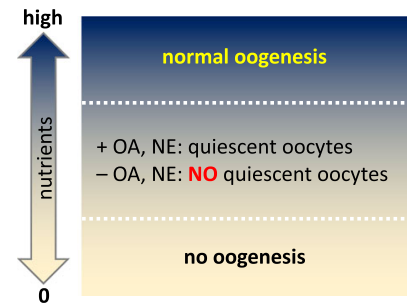

Fig. 8 The model. a The summary of oogenesis in different models shows the step and the stage where the noradrenergic input is required to maintain oocyte quiescence. Oocyte quiescence is maintained by OA. Quiescent oocytes are awakened by sperm in C. elegans and by nutrients in D. melanogaster. In

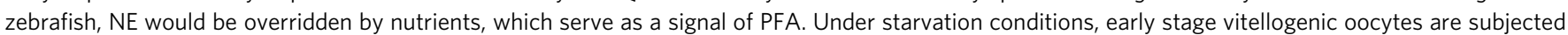
to degeneration in D. melanogaster and zebrafish. b For flies and zebrafish, oogenesis is tightly regulated by availability of food. When nutrients are completely deprived (0), oogenesis does not initiate due to lack of building blocks and energy. When nutrients are abundant (high), oogenesis progresses in full speed overriding any inhibitory signals such as NE/OA. When the nutrient level is insufficient for continuous oogenesis, NE/OA serves as a counterbalance signal to nutrients; oogenesis stops, and oocytes become quiescent waiting for the condition to be improved. 
proportion of quiescent primordial follicles in calorie-restricted animals suggests reduction of awakened primordial follicles due to a low transition rate from primordial to growing follicles. Thus, it will be intriguing to examine oogenesis of mice lacking $\mathrm{NE}$ under these starvation conditions.

\section{Methods}

Animal strains and culture conditions. Caenorhabditis elegans were routinely grown on nematode growth media (NGM) seeded with E. coli strain $\mathrm{HB} 101$ at $20^{\circ} \mathrm{C}$. The wild-type C. elegans was Bristol N2. Mutant strains used were BS553 fog-2(oz40) $V$, CB4108 fog-2(q71) V, DA1774 ser-3(ad1774) I, DA2142 fem-1(hc17ts) IV, MT9455 tbh-1(n3247) X, MT9971 nIs107 [Ptbh-1::GFP + lin-15(+)] III, MT13113 tdc-1(n3419) II. YJ249 fog-2 (q71) V; tbh-1 (n3247) $X$ was generated as follows: fog-2( $q 71)$ males were crossed to $t b h$-1(n3247) hermaphrodites. The $\mathrm{F}_{1}$ males were crossed to $t b h$ 1(n3247) hermaphrodites, and $\sim 100 \mathrm{~L} 4$ of $\mathrm{F}_{2}$ hermaphrodites were plated individually. Females of fog-2(q71) V; tbh-1(n3247) $X$, were identified by fog-2 phenotype and crossed with fog-2 males to generate males of fog-2(q71) V; tbh-1(n3247) X. NGM plates containing $20 \mathrm{mM}$ octopamine (OA) were used during crosses.

Drosophila strains, Canton-S, $w^{1118}, t b h^{n M 18} / \mathrm{FM} 7$ (a gift from Dr. Monastirioti), $t d c 2^{R O 54}$ (a gift from Dr. Hirsh), Tdc2-GAL4 (\#9313), UAS-GFP (\#4776), UAS-mCD8::GFP, and oct $\beta 2 R$ f05679 (\#18896) (from Bloomington Drosophila stock center), were used. The strains were grown on the standard cornmeal-sugar-yeast media (CSY), containing $5.2 \mathrm{~g}$ cornmeal, $11 \mathrm{~g}$ table sugar, $2.5 \mathrm{~g}$ yeast (MP Biomedicals, Solon, OH), $1.1 \mathrm{ml}$ of $20 \%$ tegosept, $0.5 \%$ propionic acid and $0.79 \mathrm{~g}$ agar per $100 \mathrm{ml}$ of $\mathrm{H}_{2} \mathrm{O}$. The stocks were maintained by setting up matings between 30 females and 30 males.

Danio rerio strains used were $d b h^{c t 806 /+}(A B / T L)$ and $\operatorname{Tg}(d b h: E G F P)$ (gifts from Dr. Prober ${ }^{26}$. Zebrafish were maintained in environmentally controlled rooms at the Bioscience and Biotechnology Center, Nagoya University, on 14-10 h light-dark cycle at $28.5^{\circ} \mathrm{C}$. Two primers to discriminate the 4 -bp size difference between wild type $(80 \mathrm{bp})$ and $d b h^{c t 806}$ mutation ( $\left.84 \mathrm{bp}\right)$ were $5^{\prime}$-TACACCATGCTGGAGCAT CCC-3' (forward) and $5^{\prime}$-AGGACTCGCTGGACGCCA-3' (reverse). Five females of $\sim 3$ months of age after gender was confirmed by the genital papilla were housed together in a tank and were subjected to starvation. Approximately 50 females of $d b h^{-/-}$and 30 females of $d b h^{+/-}$were tested.

\section{Oogenesis observation}

Caenorhabditis. The gonads of C. elegans and C. remanei were collected after dissection of the head of an animal with a needle. The gonads were fixed in ice-cold methanol for $15 \mathrm{~min}$ at $-20^{\circ} \mathrm{C}$, washed 3-5 times with PBST $(1 \times \mathrm{PBS}$, pH7.4, $0.1 \%$ Tween-20 (Sigma, P9416)) and stained with DAPI ( $1 \mu \mathrm{g} / \mathrm{ml}$ in PBST) for $5 \mathrm{~min}$ at room temperature. Approximately 50 animals were stained per strain, and the experiment was repeated at least twice. Ovulation rate were determined as the total of ovulated unfertilized oocytes in the uterus and laid on the plate at $10 \mathrm{~h}$ and at $24 \mathrm{~h}$ after the late L4 stage at room temperature (approximately at $\left.23^{\circ} \mathrm{C}\right)^{23}$. Females containing quiescent oocytes were mated with wild-type (N2) males at $20{ }^{\circ} \mathrm{C}$. To calculate \% unhatched embryo for fog- 2 and fog-2; tbh-1 (Fig. $4 \mathrm{~g}$ ), the mated females were removed, and the number of unhatched embryos were counted after $24 \mathrm{~h}$. \% unhatched embryos = Number of unhatched embryos/total laid eggs.

D. melanogaster. CS media (CSY media without yeast) were used for the protein starvation condition and CSY media for the control condition (moderately protein rich). Ten virgin females (collected within $4 \mathrm{~h}$ of eclosion) identified by light body color with greenish substance inside abdomen were transferred to a new CS media and reared at $25{ }^{\circ} \mathrm{C}$ for the indicated period of times of $0,1,1.5,2 \mathrm{dpe}$ (day post eclosion). The rearing chamber was maintained at $25^{\circ} \mathrm{C}$ with $40 \%$ humidity on a $\overline{1} 2 \mathrm{~h}: 12 \mathrm{~h} \mathrm{light}$-dark cycle. For starvation, $0.8 \%$ agar was used. OA $(5 \mathrm{mg} / \mathrm{ml}$ or $10 \mathrm{mg} / \mathrm{ml}$ ) was added while making CS media or agar.

After the indicated period, the ovaries were dissected, submerged in halocarbon oil 700 (Sigma, H8898), and observed under the microscope. The stage of an egg chamber was identified based on the described characteristics ${ }^{44}$; the stage 8 egg chambers were identified by accumulation of vitellogenin and germline encircling follicle cells and the stage 6 and 7 egg chambers were identified by the size and the shape ${ }^{44}$.

Danio rerio. Five-month-old female fish were starved for 3-6 weeks. After two weeks, they were moved to clean tanks. The ovary sample was prepared as described ${ }^{69}$. Briefly, the ovary was fixed with $4 \%$ paraformaldehyde (Sigma, P6148), embedded in a plastic casting, sectioned in $4 \mu \mathrm{m}$ thickness using a microtome (Leica RM2125) and then stained with $1 \%$ neutral red. Eight sections from ovaries of three $d b h^{+/-}$and ten sections from ovaries of four $d b h^{-1-}$ females were selected to count the number of oocytes at the indicated stage. The selected sections were separated more than $40 \mu \mathrm{m}$ from each other.

\section{Imaging}

For GFP expression. GFP-expressing strains of each animal species were mounted and observed under a fluorescence microscope (Zeiss Axio A2 Imager) or a confocal microscope (Olympus) for zebrafish.
The stained gonads of Caenorhabditis were observed under a DAPI filter and the differential interference contrast (DIC) setting using a Zeiss Axio A2 Imager with either $40 \times$ or $63 \times$ objectives. The ovaries of flies were observed using a lightfield microscopy at $10 \times$ and the ovaries of zebrafish at $5 \times$ or $3.2 \times$ magnifications. Images were acquired using Zeiss Axiovision software.

For imaging of the ovulation process a live C. elegans was observed using a $10 \times$ lens at $0 \mathrm{~h}$ and $12 \mathrm{~h}$ time points. The images of the same animals were taken using a $63 \times$ objective at the $21 \mathrm{~h}$ time point.

\section{DNA construct}

$\mathrm{P}_{\text {ceh-18 }}:$ :ser-3. The construct containing the $2.1 \mathrm{~kb}$ upstream region of ceh-18 gene was made and fused to the other construct containing the coding region and $1 \mathrm{~kb}$ downstream region of ser-3 gene. The primers for the former are $5^{\prime}$-ACACATCAGCTTA CCTGGCG- $3^{\prime}$ (forward) and $5^{\prime}$ - TCTCATCCATTCCATATTTCTTGCATTGATGA TATGTG- $3^{\prime}$ (reverse). The primers for the latter are $5^{\prime}$-AATGCAAGAAATATGGA ATGGATGAGAAATACGTTAAAC- $3^{\prime}\left(P_{\text {ceh- } 18:: s e r-3}\right.$ joining $)$ and $5^{\prime}$-GAAGGCAGC ATGTGTGCAG- $3^{\prime}$. The primers to join the two products were $5^{\prime}$-ATTTCACATCA ACACGAGTGTACG-3' (forward) and 5'-CTAAAGTTCAGTTCGTTCACGGAG-3' (reverse).

RNA interference. Bacteria-mediated feeding RNA interference (RNAi) was performed using RNAi clones from Ahringer's library. For RNAi by injection, double-stranded C. elegans $t d c-1$ and $t p h-1$ RNAs were purified and injected (with $200 \mathrm{ng} / \mathrm{ml}$ ) into the gonads of females of C. remanei at the L4 or young adult stages. Then, the progeny $\left(\mathrm{F}_{1}\right)$ were scored for the Emo phenotype.

Brood size. A brood size of each strain of C. elegans was measured by transferring a single L4 hermaphrodite to $E$. coli-seeded plates to a new plate every day. The progeny from all the plates from one hermaphrodite were counted after they grew. About eight to thirteen animals were used for each genotype.

Rescue of C. elegans OA mutants. Synchronized L4 stage C. elegans were transferred onto NGM plates with or without OA (octopamine hydrochloride, Sigma, O0250) or NE (L-Norepinephrine hydrochloride, Sigma, 74480). After treatment, the ovaries were dissected and stained with DAPI (Sigma, D9542) to examine the phenotype. DAPI stained oocytes in the gonads were observed either at $62 \mathrm{~h}$ or $89 \mathrm{~h}$ after hatching or at both time points. For the rescue, the ovaries were observed at $89 \mathrm{~h}$ after hatching in $t b h-1$ and $t d c-1$ mutants.

Antibody staining of MAPK activation. Dissected gonads from $\sim 10-15$ worms were fixed with $3 \%$ paraformaldehyde for $15 \mathrm{~min}$ at $20^{\circ} \mathrm{C}$, washed three times with $1 \times$ PBST and post-fixed in ice-cold methanol for $5 \mathrm{~min}$ at $20^{\circ} \mathrm{C}$. The fixed gonads were washed three times with $1 \times$ PBST solution and blocked for $1 \mathrm{~h}$ with $0.5 \%$ BSA in $1 \times$ PBST. After blocking, gonads were incubated with anti-MAPK-YT (Sigma, M8159, 1:500 dilution) at $4{ }^{\circ} \mathrm{C}$ for $16 \mathrm{~h}$, then incubated with an anti-mouse secondary antibody conjugated with Alexa Fluor 488 (Abcam, ab150113, 1:1,000 dilution) for $1 \mathrm{~h}$ at room temperature ${ }^{42}$.

Ovulation recording of $\boldsymbol{C}$. elegans. Young adult worms ( 0 or 1 embryo included) were anesthetized for $30 \mathrm{~min}$ in a buffer solution (M9) with $0.01 \%$ tetramisole (Sigma, L9756). Gonadal sheath contraction and ovulation were recorded for $60 \mathrm{~min}$ at 20 frames/s using a Flea3 digital camera (Point Gray Research). Contractions were counted at $1 \mathrm{~min}$ intervals.

Statistics. We examined whether all experimental groups were normally distributed using Sturges' class number and a symmetric distribution. Most groups appeared to be normally distributed, but some groups are skewed regardless of the sample size. Most OA/NE-lacking mutant groups showed much bigger variances compared with those of control groups. F test was used to determine equality of variances for two independent groups. Although samples in some groups appeared not to be normally distributed, most comparisons between control and test groups were carried out using a two-sided unpaired $t$-test with unequal (for most comparisons) or equal (for a few comparisons) variance, because sample sizes were relatively big. The data in the Result indicate mean values \pm SD. All boxplots show biological variability of each group of samples. Box-and-whisker plots show the median, mean $(X)$, interquartile range (IQR) (between the 25th and 75th). The upper whisker indicates the maxima smaller than 1.5 times IQR plus the third quartile, and the lower whisker indicates the minima larger than 1.5 times IQR minus the first quartile. The statistical tests were performed using Microsoft Excel (Microsoft, USA) and the data analysis program GraphPad Prism 9.1 and 9.2 (GraphPad Software Inc, USA). ' $n$ ' values represent biologically independent animals except for Fig. 7f-g. For 7f-g. ' $n$ ' value represents the number of independent samples. 
Reporting summary. Further information on research design is available in the Nature Research Reporting Summary linked to this article.

\section{Data availability}

Source data are provided with this paper.

Received: 13 June 2020; Accepted: 29 October 2021;

Published online: 26 November 2021

\section{References}

1. Zhang, M., Su, Y. Q., Sugiura, K., Xia, G. \& Eppig, J. J. Granulosa cell ligand NPPC and its receptor NPR2 maintain meiotic arrest in mouse oocytes. Science 330, 366-369 (2010).

2. Jaffe, L. A. \& Egbert, J. R. Regulation of mammalian oocyte meiosis by intercellular communication within the ovarian follicle. Annu. Rev. Physiol. 79, 237-260 (2017).

3. Egbert, J. R. et al. Dephosphorylation and inactivation of NPR2 guanylyl cyclase in granulosa cells contributes to the LH-induced decrease in cGMP that causes resumption of meiosis in rat oocytes. Development 141, 3594-3604 (2014).

4. Zhang, H. et al. Somatic cells initiate primordial follicle activation and govern the development of dormant oocytes in mice. Curr. Biol. 24, 2501-2508 (2014).

5. Pellatt, L. et al. Anti-Mullerian hormone reduces follicle sensitivity to folliclestimulating hormone in human granulosa cells. Fertil. Steril. 96, 1246-1251 e1241 (2011).

6. Sacchi, S. et al. The anti-Mullerian hormone (AMH) acts as a gatekeeper of ovarian steroidogenesis inhibiting the granulosa cell response to both FSH and LH. J. Assist. Reprod. Genet. 33, 95-100 (2016).

7. Durlinger, A. L. et al. Control of primordial follicle recruitment by antiMullerian hormone in the mouse ovary. Endocrinology 140, 5789-5796 (1999).

8. Edwards, R. G. Maturation in vitro of mouse, sheep, cow, pig, rhesus monkey and human ovarian oocytes. Nature 208, 349-351 (1965).

9. Eppig, J. J. \& O’Brien, M. J. Development in vitro of mouse oocytes from primordial follicles. Biol. Reprod. 54, 197-207 (1996).

10. Fortune, J. E., Cushman, R. A., Wahl, C. M. \& Kito, S. The primordial to primary follicle transition. Mol Cell Endocrinol. 163, 53-60 (2000).

11. Spence, R., Gerlach, G., Lawrence, C. \& Smith, C. The behaviour and ecology of the zebrafish, Danio rerio. Biol. Rev. Camb. Philos. Soc. 83, 13-34 (2008).

12. Selman, K., Wallace, R. A., Sarka, A. \& Qi, X. Stages of oocyte development in the zebrafish, Brachydanio rerio. J. Morphol. 218, 203-224 (1993).

13. Clelland, E. \& Peng, C. Endocrine/paracrine control of zebrafish ovarian development. Mol Cell Endocrinol. 312, 42-52 (2009).

14. Saunders, D. S., Henrich, V. C. \& Gilbert, L. I. Induction of diapause in Drosophila melanogaster: photoperiodic regulation and the impact of arrhythmic clock mutations on time measurement. Proc. Natl Acad. Sci. USA 86, 3748-3752 (1989).

15. Kubrak, O. I., Kucerova, L., Theopold, U. \& Nassel, D. R. The sleeping beauty: how reproductive diapause affects hormone signaling, metabolism, immune response and somatic maintenance in Drosophila melanogaster. PLoS ONE 9, e113051 (2014).

16. Geminard, C., Rulifson, E. J. \& Leopold, P. Remote control of insulin secretion by fat cells in Drosophila. Cell Metab. 10, 199-207 (2009).

17. Delanoue, R. et al. Drosophila insulin release is triggered by adipose Stunted ligand to brain Methuselah receptor. Science 353, 1553-1556 (2016).

18. LaFever, L. \& Drummond-Barbosa, D. Direct control of germline stem cell division and cyst growth by neural insulin in Drosophila. Science 309, 1071-1073 (2005).

19. Tatar, M. et al. A mutant Drosophila insulin receptor homolog that extends life-span and impairs neuroendocrine function. Science 292, 107-110 (2001).

20. Jindra, M., Uhlirova, M., Charles, J. P., Smykal, V. \& Hill, R. J. Genetic evidence for function of the bHLH-PAS protein Gce/Met As a juvenile hormone receptor. PLoS Genet. 11, e1005394 (2015).

21. Saunders, D. S., Richard, D. S., Applebaum, S. W., Ma, M. \& Gilbert, L. I. Photoperiodic diapause in Drosophila melanogaster involves a block to the juvenile hormone regulation of ovarian maturation. Gen. Comp. Endocrinol. 79, 174-184 (1990).

22. Schiesari, L., Andreatta, G., Kyriacou, C. P., O’Connor, M. B. \& Costa, R. The insulin-like proteins dILPs-2/5 determine diapause inducibility in drosophila. PLoS ONE 11, e0163680 (2016).

23. McCarter, J., Bartlett, B., Dang, T. \& Schedl, T. On the control of oocyte meiotic maturation and ovulation in Caenorhabditis elegans. Dev. Biol. 205, 111-128 (1999).
24. Monastirioti, M. Distinct octopamine cell population residing in the CNS abdominal ganglion controls ovulation in Drosophila melanogaster. Dev. Biol. 264, 38-49 (2003)

25. Alkema, M. J., Hunter-Ensor, M., Ringstad, N. \& Horvitz, H. R. Tyramine functions independently of octopamine in the Caenorhabditis elegans nervous system. Neuron 46, 247-260 (2005).

26. Singh, C., Oikonomou, G. \& Prober, D. A. Norepinephrine is required to promote wakefulness and for hypocretin-induced arousal in zebrafish. Elife 4, e07000 (2015)

27. Thomas, S. A. \& Palmiter, R. D. Impaired maternal behavior in mice lacking norepinephrine and epinephrine. Cell 91, 583-592 (1997).

28. Zhou, C., Rao, Y. \& Rao, Y. A subset of octopaminergic neurons are important for Drosophila aggression. Nat. Neurosci. 11, 1059-1067 (2008).

29. Horvitz, H. R., Chalfie, M., Trent, C., Sulston, J. E. \& Evans, P. D. Serotonin and octopamine in the nematode Caenorhabditis elegans. Science 216, 1012-1014 (1982).

30. Lee, H. G., Seong, C. S., Kim, Y. C., Davis, R. L. \& Han, K. A. Octopamine receptor OAMB is required for ovulation in Drosophila melanogaster. Dev. Biol. 264, 179-190 (2003).

31. Hudson, A. M., Petrella, L. N., Tanaka, A. J. \& Cooley, L. Mononuclear muscle cells in Drosophila ovaries revealed by GFP protein traps. Dev. Biol. 314, 329-340 (2008).

32. McKey, J., Bunce, C., Batchvarov, I. S., Ornitz, D. M. \& Capel, B. Neural crestderived neurons invade the ovary but not the testis during mouse gonad development. Proc. Natl Acad. Sci. USA 116, 5570-5575 (2019).

33. Iwasaki, K., McCarter, J., Francis, R. \& Schedl, T. emo-1, a Caenorhabditis elegans Sec61p gamma homologue, is required for oocyte development and ovulation. J. Cell Biol. 134, 699-714 (1996).

34. Wragg, R. T. et al. Tyramine and octopamine independently inhibit serotonin stimulated aversive behaviors in Caenorhabditis elegans through two novel amine receptors. J. Neurosci. 27, 13402-13412 (2007).

35. Govindan, J. A., Nadarajan, S., Kim, S., Starich, T. A. \& Greenstein, D. Somatic cAMP signaling regulates MSP-dependent oocyte growth and meiotic maturation in C. elegans. Development 136, 2211-2221 (2009).

36. Suo, S., Kimura, Y., Van \& Tol, H. H. Starvation induces cAMP response element-binding protein-dependent gene expression through octopamine-Gq signaling in Caenorhabditis elegans. J. Neurosci. 26, 10082-10090 (2006).

37. Rose, K. L. et al. The POU gene ceh-18 promotes gonadal sheath cell differentiation and function required for meiotic maturation and ovulation in Caenorhabditis elegans. Dev. Biol. 192, 59-77 (1997).

38. Reece-Hoyes, J. S. et al. Insight into transcription factor gene duplication from Caenorhabditis elegans Promoterome-driven expression patterns. BMC Genomics 8, 27 (2007).

39. Greenstein, D. et al. Targeted mutations in the Caenorhabditis elegans POU homeo box gene ceh-18 cause defects in oocyte cell cycle arrest, gonad migration, and epidermal differentiation. Genes Dev. 8, 1935-1948 (1994).

40. Kimble, J., Edgar, L. \& Hirsh, D. Specification of male development in Caenorhabditis elegans: the fem genes. Dev. Biol. 105, 234-239 (1984).

41. Schedl, T. \& Kimble, J. fog-2, a germ-line-specific sex determination gene required for hermaphrodite spermatogenesis in Caenorhabditis elegans. Genetics 119, 43-61 (1988).

42. Miller, M. A. et al. A sperm cytoskeletal protein that signals oocyte meiotic maturation and ovulation. Science 291, 2144-2147 (2001).

43. Castaneda, P. G., Cecchetelli, A. D., Pettit, H. N. \& Cram, E. J. Galpha/GSA-1 works upstream of $\mathrm{PKA} / \mathrm{KIN}-1$ to regulate calcium signaling and contractility in the Caenorhabditis elegans spermatheca. PLoS Genet. 16, e1008644 (2020).

44. King, R. C. The meiotic behavior of the Drosophila oocyte. Int. Rev. Cytol. 28, 125-168 (1970).

45. Drummond-Barbosa, D. \& Spradling, A. C. Stem cells and their progeny respond to nutritional changes during Drosophila oogenesis. Dev. Biol. 231, 265-278 (2001).

46. Shimada, Y., Burn, K. M., Niwa, R. \& Cooley, L. Reversible response of protein localization and microtubule organization to nutrient stress during Drosophila early oogenesis. Dev. Biol. 355, 250-262 (2011).

47. Burn, K. M. et al. Somatic insulin signaling regulates a germline starvation response in Drosophila egg chambers. Dev. Biol. 398, 206-217 (2015).

48. Piper, M. D. et al. A holidic medium for Drosophila melanogaster. Nat Methods 11, 100-105 (2014).

49. Jouandin, P., Ghiglione, C. \& Noselli, S. Starvation induces FoxO-dependent mitotic-to-endocycle switch pausing during Drosophila oogenesis. Development 141, 3013-3021 (2014).

50. Monastirioti, M., Linn, C. E. Jr. \& White, K. Characterization of Drosophila tyramine beta-hydroxylase gene and isolation of mutant flies lacking octopamine. J. Neurosci. 16, 3900-3911 (1996).

51. Maqueira, B., Chatwin, H. \& Evans, P. D. Identification and characterization of a novel family of Drosophila beta-adrenergic-like octopamine G-protein coupled receptors. J. Neurochem. 94, 547-560 (2005). 
52. Ohhara, Y., Kayashima, Y., Hayashi, Y., Kobayashi, S. \& YamakawaKobayashi, K. Expression of beta-adrenergic-like octopamine receptors during Drosophila development. Zoolog. Sci. 29, 83-89 (2012).

53. Crocker, A., Shahidullah, M., Levitan, I. B. \& Sehgal, A. Identification of a neural circuit that underlies the effects of octopamine on sleep:wake behavior. Neuron 65, 670-681 (2010).

54. Govindan, J. A., Cheng, H. \& Harris, J. E. Greenstein D. Galphao/i and Galphas signaling function in parallel with the MSP/Eph receptor to control meiotic diapause in C. elegans. Curr. Biol. 16, 1257-1268 (2006).

55. Yang, Z. et al. Octopamine mediates starvation-induced hyperactivity in adult Drosophila. Proc. Natl Acad. Sci. USA 112, 5219-5224 (2015).

56. Corrales-Carvajal V. M., Faisal A. A. \& Ribeiro C. Internal states drive nutrient homeostasis by modulating exploration-exploitation trade-off. Elife 5, e19920 (2016)

57. Koon, A. C. et al. Autoregulatory and paracrine control of synaptic and behavioral plasticity by octopaminergic signaling. Nat. Neurosci. 14, 190-199 (2011).

58. Middleton, C. A. et al. Neuromuscular organization and aminergic modulation of contractions in the Drosophila ovary. BMC Biol. 4, 17 (2006).

59. Lara, H. E. et al. Changes in sympathetic nerve activity of the mammalian ovary during a normal estrous cycle and in polycystic ovary syndrome: Studies on norepinephrine release. Microsc. Res. Tech. 59, 495-502 (2002).

60. Lawrence, I. E. Jr. \& Burden, H. W. The origin of the extrinsic adrenergic innervation to the rat ovary. Anat. Rec. 196, 51-59 (1980).

61. Goldstein, J. L. et al. Surviving starvation: essential role of the ghrelin-growth hormone axis. Cold Spring Harb. Symp. Quant. Biol. 76, 121-127 (2011).

62. Tao, J., Ma, Y. C., Yang, Z. S., Zou, C. G. \& Zhang, K. Q. Octopamine connects nutrient cues to lipid metabolism upon nutrient deprivation. Sci. Adv. 2, e1501372 (2016).

63. Dowen, R. H. CEH-60/PBX and UNC-62/MEIS coordinate a metabolic switch that supports reproduction in C. elegans. Dev. Cell 49, 235-250 e237 (2019).

64. Greiner, M., Paredes, A., Araya, V. \& Lara, H. E. Role of stress and sympathetic innervation in the development of polycystic ovary syndrome. Endocrine 28, 319-324 (2005).

65. Sverrisdottir, Y. B., Mogren, T., Kataoka, J., Janson, P. O. \& Stener-Victorin, E. Is polycystic ovary syndrome associated with high sympathetic nerve activity and size at birth? Am. J. Physiol. Endocrinol. Metab. 294, E576-E581 (2008).

66. Wulff, C., Wiegand, S. J., Saunders, P. T., Scobie, G. A. \& Fraser, H. M. Angiogenesis during follicular development in the primate and its inhibition by treatment with truncated Flt-1-Fc (vascular endothelial growth factor Trap(A40). Endocrinology 142, 3244-3254 (2001).

67. Lintern-Moore, S. \& Everitt, A. V. The effect of restricted food intake on the size and composition of the ovarian follicle population in the Wistar rat. Biol. Reprod. 19, 688-691 (1978).

68. Nelson, J. F., Gosden, R. G. \& Felicio, L. S. Effect of dietary restriction on estrous cyclicity and follicular reserves in aging C57BL/6J mice. Biol. Reprod. 32, 515--522 (1985).

69. Nakamura, S. et al. Identification and lineage tracing of two populations of somatic gonadal precursors in medaka embryos. Dev. Biol. 295, 678-688 (2006).

\section{Acknowledgements}

We thank Drs. Monastirioti, Hirsh, Green, Kamikouchi, and Prober as well as the Caenorhabditis Genetics Center (supported by NIH grant P40 OD010440) and the Bloomington fly stock for strains. We thank Drs. Avery and Strauss for invaluable discussions, Drs. Gallagher and Wyler for critical reading of this manuscript, Dr. Tanaka, Y. Kazuki, Dr. Clam, A. Danielle, B. Lee, Y. Lee, Dr. Padmanabha, W. Seo, H. Kim, S.
Ahn. and N. Suryawinata for technical assistance. We thank the members of the Hibi lab and the Min lab for their help in maintaining animals. This work was supported by Inha University and Basic Science Research Program through the National Research Foundation of Korea funded by the Ministry of Education (2017R1D1A1B03036182) (J.K.), the Korea Institute of Toxicology (KK-2011-03) and National Research Foundation of Korea (NRF) (2021R1F1A1045599) (M.H.), JSPS KAKENHI JP18H02448 and CREST Japan Science and Technology Agency (JST) JPMJCR1753 (M.H.), by Virginia Commonwealth University and Nagoya University (Y-J.Y.).

\section{Author contributions}

Y.Y. conceived the idea and supervised the project. J.K. conceived the idea, performed the experiments for Figs. 1, 3-7. M.H. performed the experiments for Figs. 2 and 3. M.H. performed the experiments for Fig. 7 and supervised the zebrafish study. J.K. and Y.Y. wrote the manuscript with input from M.H. and M.H.

\section{Competing interests}

The authors declare no competing interests.

\section{Ethics statement}

The animal work in this study was approved by the Nagoya University Animal Experiment Committee and was conducted in accordance with the "Regulations on Animal Experiments in Nagoya University" and "Guidelines for Proper Conduct of Animal Experiments (Science Council of Japan)”.

\section{Additional information}

Supplementary information The online version contains supplementary material available at https://doi.org/10.1038/s41467-021-26945-x.

Correspondence and requests for materials should be addressed to Young-Jai You.

Peer review information Nature Communications thanks the anonymous reviewer(s) for their contribution to the peer review of this work. Peer reviewer reports are available.

Reprints and permission information is available at http://www.nature.com/reprints

Publisher's note Springer Nature remains neutral with regard to jurisdictional claims in published maps and institutional affiliations.

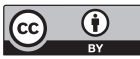

Open Access This article is licensed under a Creative Commons Attribution 4.0 International License, which permits use, sharing, adaptation, distribution and reproduction in any medium or format, as long as you give appropriate credit to the original author(s) and the source, provide a link to the Creative Commons license, and indicate if changes were made. The images or other third party material in this article are included in the article's Creative Commons license, unless indicated otherwise in a credit line to the material. If material is not included in the article's Creative Commons license and your intended use is not permitted by statutory regulation or exceeds the permitted use, you will need to obtain permission directly from the copyright holder. To view a copy of this license, visit http://creativecommons.org/ licenses/by/4.0/.

This is a U.S. Government work and not under copyright protection in the US; foreign copyright protection may apply 2021 